\title{
An Arlequin-based method to couple molecular dynamics and finite element simulations of amorphous polymers and nanocomposites
}

\author{
S. Pfaller* M. Rahimi ${ }^{\dagger}$ \\ G. Possart* \\ P. Steinmann* \\ F. Müller-Plathe ${ }^{\dagger}$ \\ M. C. Böhm ${ }^{\dagger}$
}

March 26, 2013

\begin{abstract}
A new simulation technique is introduced to couple a flexible particle domain as encountered in soft-matter systems and a continuum which is solved by the Finite Element (FE) method. The particle domain is simulated by a molecular dynamics (MD) method in coarse grained (CG) representation. On the basis of computational experiences from a previous study, a staggered coupling procedure has been chosen. The proposed MD-FE coupling approximates the continuum as a static region while the MD particle space is treated as a dynamical ensemble. The information transfer between MD and FE domains is realized by a coupling region which contains, in particular, additional auxiliary particles, so-called anchor points. Each anchor point is harmonically bonded to a standard MD particle in the coupling region. This type of interaction offers a straightforward access to force gradients at the anchor points that are required in the developed hybrid approach. Time-averaged forces and force gradients from the MD domain are transmitted to the continuum. A static coupling procedure, based on the Arlequin framework, between the FE domain and the anchor points provides new anchor point positions in the MD-FE coupling region. The capability of the new simulation procedure has been quantified for an atactic polystyrene (PS) sample and for a PS-silica nanocomposite, both simulated in CG representation. Numerical data are given in the linear elastic regime which is conserved up to $3 \%$ strain. The convergence of the MD-FE coupling procedure has been demonstrated for quantities such as reaction forces or the Cauchy stress which have been determined both in the bare FE domain and in the coupled system. Possible applications of the hybrid method are shortly mentioned.
\end{abstract}

Keywords: particle-continuum coupling, multiscale modeling, bridging domain method, domain decomposition, Lagrange multipliers, nanocomposites

\section{Introduction and outline}

Continuum mechanics, a field-based theory that can be treated numerically by the widely used Finite Element (FE) method, is a very powerful tool to simulate the macroscopic mechanical behaviour of e.g. solids. Unfortunately, it is less suited in cases where molecular or even atomistic details become important to capture the phenomena of interest. On the other hand, such particle-based information becomes accessible either by Monte Carlo (MC) or molecular dynamics (MD) calculations. The application of MC or MD simulations for macroscale problems, however, is computationally prohibitive due to the large number of particles required and the corresponding degrees of freedom. Typical system sizes in MD calculations are in the range of nanometers, while typical orders of magnitude in the time steps amount to femtoseconds, which allow simulation times of some nanoseconds. Compared to the time scales and system sizes relevant in engineering problems of continuum mechanics, the time and length scales considered in MD simulations are, thus, orders of magnitude smaller. The advantages of

\footnotetext{
*Chair of Applied Mechanics, University of Erlangen-Nuremberg, Egerlandstrasse 5, 91058 Erlangen, Germany, sebastian.pfaller@ltm.uni-erlangen.de,gunnar.possart@ltm.uni-erlangen.de,paul.steinmann@ltm.uni-erlangen.de

${ }^{\dagger}$ Eduard-Zintl-Institut für Anorganische und Physikalische Chemie and Centre of Smart Interfaces, Technische Universität Darmstadt, Petersenstr. 20, 64287 Darmstadt, Germany, Tel.: +49 (0)6151 16 6523, Fax: +49 (0)6151 16 6526,m.rahimi@theo.chemie.tu-darmstadt.de, boehm@theo.chemie.tu-darmstadt.de, f.mueller-plathe@theo.chemie.tudarmstadt.de
} 
both theoretical tools can be combined within hybrid techniques which allow the coupling of a particle description to a continuum one or the coupling of particle domains with different resolutions. Such methods are particularly useful under conditions where molecular details are relevant only in smaller spatial regions of the studied sample such as solid-fluid or solid-polymer interfaces, while particle properties are not required in the remaining regions which have bulk behaviour. In such systems the efficiency of continuum mechanics can be combined with the accuracy of MD simulations.

In the past years an increasing number of publications have proposed combinations of particle-based models with field-based models in hybrid schemes. Within this contribution, we will concentrate on hybrid schemes which are based on a spatial decomposition into a particle region and a much larger continuum. Unlike this, so-called parameter inheritance schemes have become more popular since they can be formulated more easily. These methods allow computing material parameters at the molecular level and use them as input in the subsequent continuum simulations. Quite similarly, single-chain-inmean-field models have been developed, cf. e.g. [1, 2], and [3]. Hybrid approaches can be divided into a group for hydrodynamics problems and one for structural mechanics problems. We will focus here on the second type, which often deal with the failure of crystalline systems. However, there are only few adaptions to amorphous systems, which we will concentrate on in this contribution. Well-known examples are the quasi-continuum methods as they were introduced and employed e.g. in [4, [5], and 6. Here, selected particles are moved by an affine deformation of the elements since they are attached to FE nodes or edges.

Beyond these kinds of coupling methods, extensions towards amorphous materials have been made by the so-called bridging domain method introduced by Belytschko and co-workers e.g. in [7] and [8], employed in modelling studies of carbon nanotubes as well as other systems, cf. 9] and [10. Quite similar, Ben Dhia and Rateau developed a hybrid scheme called the Arlequin method, cf. [11, [12, and [13. Originally used as a tool to couple FE domains of different resolutions, the Arlequin method has been employed later in hybrid simulations of atomistic and continuum models, cf. e.g. [14].

The Arlequin method considers a pure particle region without any underlying FE description, i.e. it does not require the particles to be arranged in a lattice. Instead, a handshake region is introduced in which the FE domain and the particle region overlap. Here the deformation of the continuum and the displacements of the particles are required to match in a weak sense. Thus, it is a suitable candidate to model amorphous materials like polymers. Although there is already a small number of publications available dealing with generic polymer models, by e.g. the group of Prudhomme, cf. 15] and [16, no procedure has been described yet that allows a coupling between an FE domain and a particle system computed with the commonly used MD tools at finite temperature.

As mentioned above, engineering problems captured by continuum mechanics and microscopic considerations treated by particle-based methods differ tremendously in time and length scales. Thus, both aspects have to be considered carefully here: on the one hand, the spatial scale bridging as introduced here seems to be feasible. Its formulation is supported by the publications on static continuum-particle coupling procedures discussed above. On the other hand, the coupling of time scales that are orders of magnitude different is a very crucial part of any coupling scheme, and it has not been solved yet. In this context, we refer to the group of de Borst and co-workers, which has investigated the spatial and temporal coupling and discussed its advantages and drawbacks in [17.

In this contribution, we have developed a new hybrid technique for polymers to couple a particle domain to a continuum. In order to avoid conceptual problems arising from the different time scales in the respective regions, the continuum is treated purely static while only the particle domain is computed dynamically. This seems to be reasonable due to the large difference between the dynamics on the macroscale and that of the particles: any time-dependent processes on a scale relevant for engineering problems would be quasi-static compared to the dynamics at the level of particles. In the following, we will sketch the main components of the coupling procedure and point to the corresponding sections where these aspects will be discussed in detail.

First, the MD domain treated in the present coupling scheme is large in comparison to dimensions usually encountered in atomistic MD simulations. Therefore the MD region has to be treated by a coarse grained (CG) technique [18, 19, 20, which reduces the degrees of freedom by grouping a number of atoms together into so-called superatoms or CG beads. The CG potentials used in the present work have been derived by iterative Boltzmann inversion (IBI) of atomistic potentials [21. Quite generally the CG mapping offers access to simulations which are computationally unfeasible in a purely atomistic model. To perform coupled MD-FE simulations the conventional periodic boundary conditions (PBCs) had to be replaced by nonperiodic stochastic ones (SBCs) 22. In the boundary region of the developed coupling scheme we have defined a set of auxiliary particles, so-called anchor 
points. They are harmonically coupled to the MD particles. The anchor points form a set of fixed particles without interaction between them that do not move during the MD equilibration procedure. Hence, they can be coupled to a static continuum as mediators between the different domains. This static coupling will be realized with the help of the Arlequin method mentioned above.

Thus, the spatial set-up of our hybrid method consists of three main regions as sketched in Figure 1;

1. a pure particle domain $\Omega^{d}$;

2. a bridging domain $\Omega^{b}$ where the particle region overlaps with the continuum. This region contains the anchor points;

3. a pure continuum $\Omega^{c}$, discretized by finite elements.

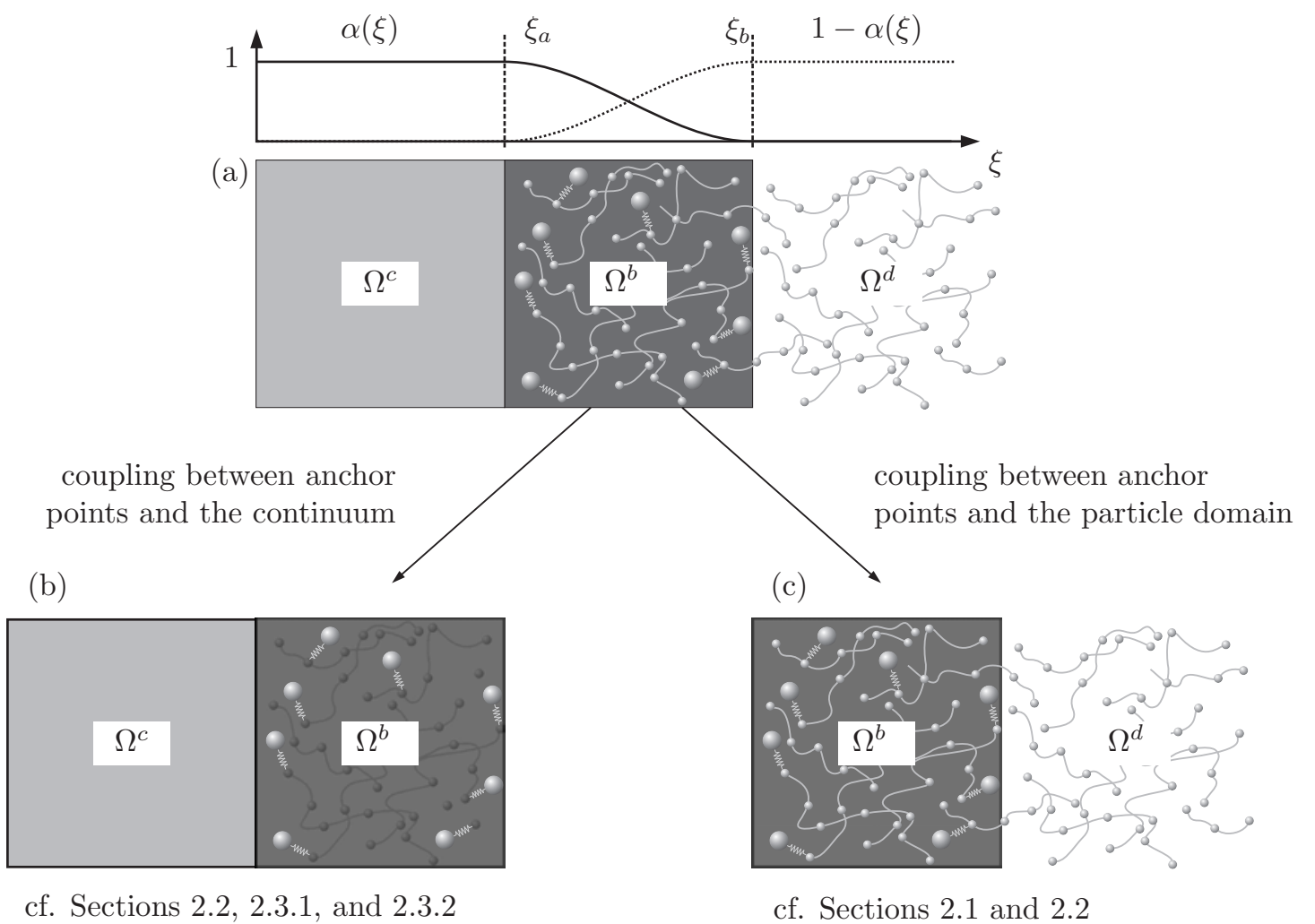

Figure 1: Spatial coupling: continuum $\Omega^{c}$, bridging domain $\Omega^{b}$, and particle domain $\Omega^{d}$; small spheres: MD particles, large spheres: anchor points; the weighting factor $\alpha(\xi)$ as well the variable $\xi$ will be explained later; the complete spatial setup (a) is separated into a coupling between the anchor points and the continuum (b) and into a coupling between the anchor points and the particle domain (c).

The scale bridging procedure proposed here is subdivided into a static coupling between the anchor points and the continuum (cf. part (b) in Figure 1) and into its counterpart which tethers the anchor points to the particle domain (cf. part (c) in Figure 1). We will subsequently discuss the respective schemes as follows:

- Sections 2.1 and 2.2 dynamic coupling between anchor points and particles as well as necessary modifications of the particle domain

- Sections 2.2 2.3.1 and 2.3.2 static coupling between anchor points and the continuum, introduction of the Arlequin method

It has to be remarked here that, due to the subdivision into a static and a dynamic scheme, the usual tools to solve the continuum and the particle domain, i.e. the conventional FE and MD procedures, can be applied with rather small modifications. Thus, algorithms and machines highly specialized for the respective tools can be employed which is important especially with respect to the very time consuming MD simulations. 
In order to embed both coupling schemes into a single computational procedure, a staggered algorithm as described below has been developed. Within a previous contribution 23, a systematic overview of staggered solution schemes has been given for the hypothetical case of a one-dimensional coupling between a continuum solved with FE and a particle domain mapped by MD. This was aiming at a sound understanding and possible solution of the problems which may appear in such couplings. The results of this examination are used and extended in the present work to couple more realistic systems, i.e. three-dimensional polymer samples as well as nanocomposites containing a polymer component. To render this possible, we had to introduce auxiliary particles, so-called anchor points, which realize a transfer of information between continuum and particle domain. This ansatz for three-dimensional amorphous polymer and composite samples is by no means trivial, at least if compared to crystalline solids, for which hybrid schemes are easier to implement since the particles are spatially confined to a lattice. For the description of the continuum, the theoretical derivations are quite similar to those in [23], whereas the treatment of the particle domain had to be modified significantly. In order to provide a comprehensive description of the methodology, all relevant aspects are recalled subsequently.

Without going into detail, only the conceptual ideas of the staggered scheme are discussed here, for a detailed description we refer to Section 3 and Figure 5. After applying a load to the undeformed continuum, the resulting deformation is transferred via the bridging region and anchor points to the MD domain which is thus distorted. Consequently, a new equilibrium has to be computed for the MD domain. This leads to reaction forces acting on the anchor points and, therefore, also on the continuum. Then, the FE domain has to be re-equilibrated, too, and takes a new configuration. This causes another distortion of the MD domain which implies that the coupling procedure has to be continued until the coupled system takes its global energy minimum.

Since the present article intends to introduce and demonstrate a new hybrid method, it is applied to an established system. We have chosen atactic polystyrene (PS) as well as a PS-silica nanocomposite in CG representation as model systems. The mapping scheme of Qian et al. 24 is used for the coarse graining of the polymer, i.e. each molecular repeat unit is described by a single bead. In atactic PS two different absolute configurations are found in the molecular fragments; they cause two types of CG beads which differ in their potential. In the coarse graining of the silica nanoparticle each $\mathrm{SiO}_{2}$ unit is mapped by a CG bead. Details of the CG procedure can be found in a recent paper of Ghanbari et al. 25]. For all MD simulations the in-house computer code IBiSCO 26] working with tabulated potentials is employed.

\section{Modeling foundations}

In this section, a short overview of the basics of continuum mechanical and particle-based modeling is given. Additionally, some aspects of coupling different domains are discussed. Beginning with the particle system and its extension with anchor points, the coupling between anchor points and the continuum, which is realized by the Arlequin method, is presented.

\subsection{Particle system and its dynamic coupling to anchor points}

Since the particle domain is surrounded by a continuum, PBCs as the usual procedure to avoid surface effects in MD cannot be used. Instead, non-periodic boundary conditions have been introduced, which satisfy the following major requirements:

1. it has to be ensured that artefacts due to the dynamics are kept to a minimum;

2. a transfer of information (forces and deformations) between the continuum and the particle region has to be realized.

To this end, a modified MD tool based on stochastic boundary conditions (SBC) has been developed. It was described in detail in [22, where it also had been applied to polystyrene. We therefore give only a brief description of its features here.

Two regions are defining the coupling layer between particle and continuum domain, cf. Figure 2, on the one hand the effective boundary region, on the other hand a dissipative particle dynamics (DPD) region, cf. e.g. 27], 26], and [28]. Using SBCs, the actual MD domain is embedded into a DPD region, which itself is enclosed by the effective boundary region. To minimize the surface effects at the interface to the continuum, stochastic dynamics is employed. The particles in the DPD region as well as in the effective boundary region move according to the DPD equations of motion, which we will not comment 


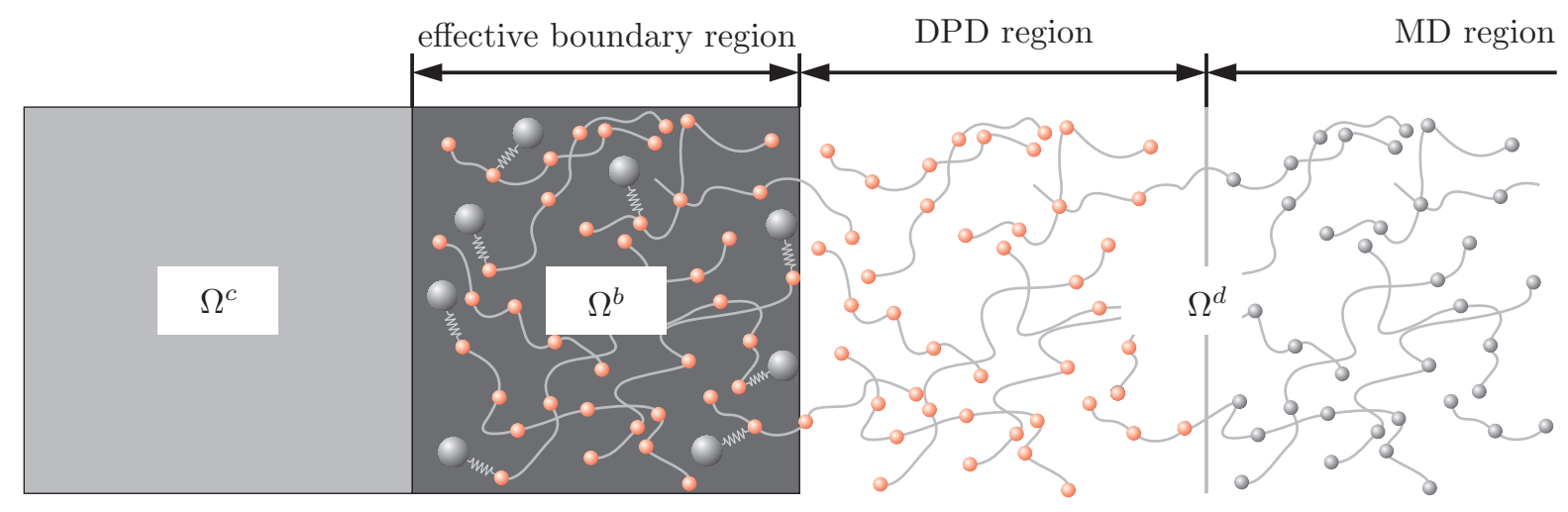

Figure 2: Spatial coupling setup with stochastic boundary conditions, consisting of continuum $\Omega^{c}$, bridging domain $\Omega^{b}$ and particle domain $\Omega^{d}$ as described in Section 1. Large spheres are anchor points while small grey spheres are particles moved according to MD equations of motion. Small red spheres are particles following DPD equations of motion, both within the effective boundary and the DPD region.

on in detail here. The stochastic dynamics pretends a large number of particles outside the MD region and acts as an external bath that is able to control the temperature of the MD region. The effective boundary region overlaps with the continuum and coincides with the bridging domain $\Omega^{b}$, which, in turn, realizes the static coupling between the particle domain and the continuum. In this region, anchor points are introduced as auxiliary particles which do not move during the MD equilibration. They confine the designated MD region, i.e. they prevent its particles from escaping. Furthermore, the anchor points exert a pressure to define a certain thermodynamic state in the MD region. Each anchor point $I$ is connected to an individual MD particle via the following harmonic potential:

$$
E_{M D I}^{i n t}=\frac{1}{2} k_{I}\left|\mathbf{R}_{I}^{M S}-\mathbf{r}_{I}^{M D}\right|^{2}
$$

In (11), $k_{I}$ denotes the force constant, $\mathbf{R}_{I}^{M S}$ is the position of the anchor point while $\mathbf{r}_{I}^{M D}$ is the current position of the MD particle it is tethered to. The subsequent section explains the terminology used here in more detail. To avoid perturbations on the particle domain due to the anchor points, the stiffness $k_{I}$ of the connection between these auxiliary units and the MD particles should be similar to that of the unperturbed particle domain.

At first sight it may seem strange to introduce anchor points for the transfer of information instead of assigning this function to an appropriate subset of the MD particles. During the solution of the MD equations of motion, this subset of particles would have to be treated differently than the conventional MD particles. Firstly, to keep these particles spatially fixed in MD simulations is not straightforwardly possible. Though, this fixation is indispensable for the stochastic boundary conditions since the continuum is statically coupled to the particle domain via the Arlequin method. Even more important is the fact, that the Arlequin framework relies on second order optimization methods to efficiently equilibrate the coupled system. These methods inter alia require the gradients of the forces exerted by the interior on the subset of particles that confine the MD region. In coarse grained simulations as used here, all interactions between MD particles are prescribed by tabulated potentials, which prohibits the analytical determination of force gradients. From a computational point of view, it is furthermore too expensive to numerically determine the force gradients. Due to the dynamics, time averaging is required already to compute the necessary force values. The above arguments justify the introduction of anchor points. Concerning their coupling to the conventional MD particles, we here have chosen the simplest (harmonic) potential, i.e. the required gradients coincide to the force constant. This choice is reasonable since most of the tabulated coarse grained potentials themselves are based on harmonic approximations and have been obtained via Inverse Boltzmann Inversion 21.

The anchor points may, furthermore, be interpreted as penalty terms controlling the coupling strength between continuum and particle domain. If $k_{I}$ is chosen small, the coupling is very weak, i.e. the MD domain is hardly affected by the deformations of the continuum. In case $k_{I}$ approaches infinity, the coupling becomes very strong, i.e. the displacements of the anchor points are almost the same as those of the attached MD particles. The MD simulation will then require much shorter time steps, which, 
in turn, leads to very high computation times. Although more extended investigations are required in this regard, it has been observed already that $k_{I}$ has to be chosen material-dependent and as large as possible. It furthermore seems to be reasonable 22] to choose $k_{I}$ similar to the bond force constant used in the pure MD domain. For simplicity, all $k_{I}$ are set to the same value here. As stated above, the anchor points are coupled to the continuum via the Arlequin method. They act as information transmitters between continuum and particle domain: The particle domain exerts forces on the anchor points, which act on the continuum similar to a Neumann boundary condition. Vice versa, the continuum is deforming the particle domain by modifying the positions of anchor points. An additional benefit of using anchor points is the fact that their number can be chosen much smaller than that of the conventional MD particles. This leads to a significant reduction of the degrees of freedom that have to be coupled to the continuum. The details will be given below.

\subsection{Interaction between anchor points and superatoms}

In order to prepare the coupling of anchor points to the continuum, the interaction between anchor points and superatoms as discussed in Section 2.1 are described more in detail. Here, the focus is on the change of anchor point positions due to the continuum deformation. Without knowing yet the relation between anchor point displacements and the continuous displacement field, cf. Section 2.3.2, the change of anchor point positions with respect to the positions of the MD particles is investigated. Please note that during the equilibration of the continuum the MD particles remain fixed which will be presented in detail in Section 3. In order to distinguish between anchor points and superatoms, we use the following terminology: variables referring to superatoms, i.e. "real" MD particles, are denoted by the superscript "MD", whereas variables describing anchor point values are labelled by the superscript "MS", which marks them as static particles, being part of a "molecular statics" region.

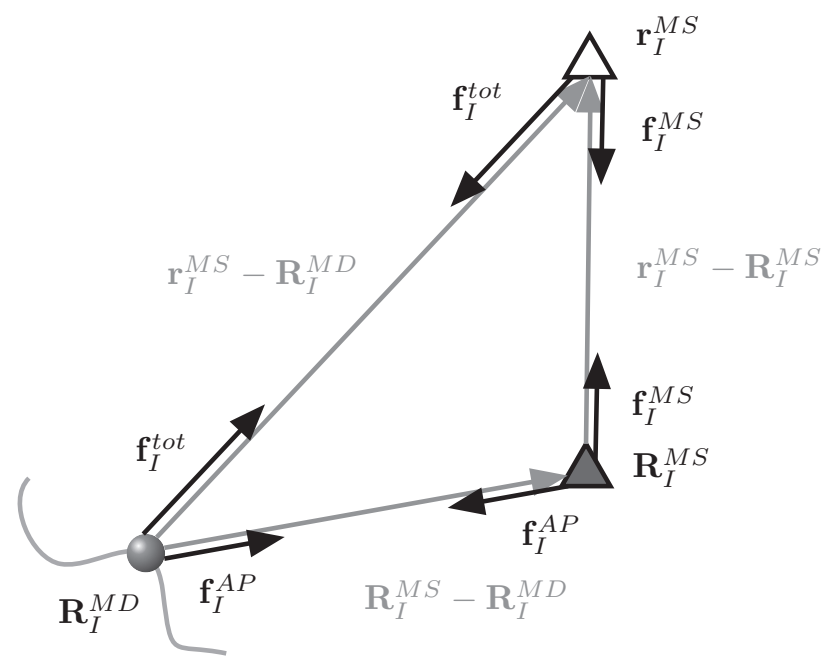

Figure 3: Interaction between superatom $I$ (position $\mathbf{R}_{I}^{M D}$ ) and its associated anchor point (initial position $\mathbf{R}_{I}^{M S}$, current position after deformation of the continuum $\mathbf{r}_{I}^{M S}$ ); force $\mathbf{f}_{I}^{A P}$ between superatom and anchor point before deformation, force $\mathbf{f}_{I}^{\text {tot }}$ between superatom and anchor point after deformation, and $\mathbf{f}_{I}^{M S}=\mathbf{f}_{I}^{t o t}-\mathbf{f}_{I}^{A P}$

Figure 3 displays the interaction of an arbitrary MD particle (position vector $\mathbf{R}_{I}^{M D}$ ) and the anchor point $I$ it is tethered to (initial position $\mathbf{R}_{I}^{M S}$, position after deformation $\mathbf{r}_{I}^{M S}$ ). During the MD equilibration, the positions of anchor points are spatially fixed while the MD particles are allowed to move according to their interactions with other MD particles. Opposite to this, we focus here on the equilibration of the continuum, where the positions of the MD particles remain fixed and the anchor points are moved according to the deformation of the continuum.

The interaction between an anchor point and its MD coupling partner is realized by a harmonic interaction potential

$$
E_{M S I}^{i n t}=\frac{1}{2} k_{I}\left|\mathbf{r}_{I}^{M S}-\mathbf{R}_{I}^{M D}\right|^{2}
$$

which is similar to (10), but formulated from the continuum point of view. Here, the anchor points are moved according to the continuum deformation whereby the superatoms remain fixed. The derivative 
of (2) with respect to the current anchor point position, i.e. the section force, can be written as

$$
\begin{aligned}
\mathbf{f}_{I}^{t o t} & =-\frac{\partial E_{M S I}^{i n t}}{\partial \mathbf{r}_{I}^{M S}}=-k_{I}\left[\mathbf{r}_{I}^{M S}-\mathbf{R}_{I}^{M D}\right] \\
& =-k_{I}\left[\mathbf{r}_{I}^{M S}-\mathbf{R}_{I}^{M S}\right]-k_{I}\left[\mathbf{R}_{I}^{M S}-\mathbf{R}_{I}^{M D}\right] \\
& =\mathbf{f}_{I}^{M S}+\mathbf{f}_{I}^{A P} .
\end{aligned}
$$

In (3), the force $\mathbf{f}_{I}^{\text {tot }}$ acting on the anchor point due to its interaction with the related MD particle, is separated into $\mathbf{f}_{I}^{A P} \neq \mathbf{f}_{I}^{A P}\left(\mathbf{r}_{I}^{M S}\right)$ and $\mathbf{f}_{I}^{M S}=\mathbf{f}_{I}^{M S}\left(\mathbf{r}_{I}^{M S}, \mathbf{R}_{I}^{M S}\right)$ which will be used in Section 3 when setting up the detailed coupling algorithm.

Furthermore, $\mathbf{f}_{I}^{M S}$ can be rewritten as

$$
\begin{aligned}
\mathbf{f}_{I}^{M S} & =-\mathbf{K}_{I}^{A P} \cdot\left[\mathbf{r}_{I}^{M S}-\mathbf{R}_{I}^{M S}\right]=-\mathbf{K}_{I}^{A P} \cdot \mathbf{w}_{I} \quad \text { with } \\
\mathbf{K}_{I}^{A P} & =\left[\begin{array}{ccc}
k_{I} & 0 & 0 \\
0 & k_{I} & 0 \\
0 & 0 & k_{I}
\end{array}\right],
\end{aligned}
$$

where $\mathbf{w}_{I}=\mathbf{r}_{I}^{M S}-\mathbf{R}_{I}^{M S}$ denotes the displacement of the anchor point and $\mathbf{K}_{I}^{A P}$ is the diagonal matrix of force constants. Since there is no interaction with other anchor points considered, the force acting on each anchor point is only a function of its relative position to the respective superatom.

\subsection{Continuum and its static coupling to anchor points}

In this section, the static coupling between the continuum and the anchor points is described. First of all, the basics of continuum mechanics will be sketched, followed by the introduction of the Arlequin method. After that, both, the continuum formulations as well as the results from Section 2.2 will be embedded into the Arlequin framework and the spatial discretization of the static coupling will be discussed.

\subsubsection{Continuum modeling}

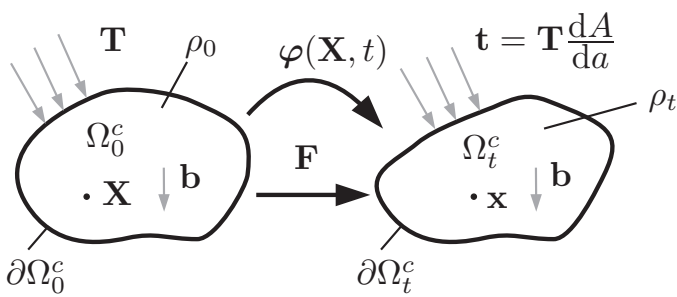

Figure 4: Continuum mechanical setting: deformation map $\varphi$ from material configuration $\Omega_{0}^{c}$ to spatial configuration $\Omega_{t}^{c}$; quantities are defined in the text

In continuum mechanics, the undeformed (material) configuration $\Omega_{0}^{c}$ of a continuous body is mapped to the deformed (spatial) configuration $\Omega_{t}^{c}$ by a vector-valued function

$$
\varphi: \mathbf{X} \mapsto \mathbf{X}=\varphi(\mathrm{X}, t)
$$

which is sketched in Figure 4. A point in $\Omega_{0}^{c}$ is identified by its position vector $\mathbf{X}$, whereas $\mathbf{x}$ denotes the same point in the deformed configuration. To describe strains in the material, the deformation gradient

$$
\mathbf{F}=\frac{\partial \varphi}{\partial \mathbf{X}}=\frac{\partial}{\partial \mathbf{X}}[\mathbf{X}+\mathbf{u}]=\mathbf{I}+\frac{\partial \mathbf{u}}{\partial \mathbf{X}}
$$

is introduced, together with displacement vector $\mathbf{u}=\mathbf{x}-\mathbf{X}$ and second order identity $\mathbf{I}$.

\subsubsection{Arlequin method}

As described in Sections 2.1] and 2.2. the exchange of information between continuum and particle domain is realized by anchor points. Since these are not moved within the MD calculations, their 
coupling to the continuum is of a static nature. We thus can employ the "Arlequin" approach as introduced by Ben Dhia and Rateau, cf. [11, [12] and [13, for this coupling.

The Arlequin framework blends the energies of particles and continuum via a weighting factor $\alpha$. Furthermore, a kinematic constraint is introduced to enforce a match of particle and continuum displacements.

The coupling is sketched in Figure 1 the continuum domain $\Omega^{c}$ is coupled to the particle domain $\Omega^{d}$ via a bridging domain $\Omega^{b}$. Since we focus here on the static coupling of the anchor points to the continuum, the DPD region as defined in Section 2.1 is not considered in the following discussion.

In order to weight the energies in the respective domains, we introduce a weighting factor $\alpha(\xi)$ with $\xi=\xi(\mathbf{X})$ in the continuum domain and $\xi=\xi\left(\mathbf{R}_{I}\right)$ in the particle domain. The following conditions have to hold:

$$
\alpha(\xi)\left\{\begin{array}{ll}
=1 & \text { in } \Omega^{c} \backslash \Omega^{b} \\
\in[0,1] & \text { in } \Omega^{b} \\
=0 & \text { in } \Omega^{d} \backslash \Omega^{b}
\end{array} .\right.
$$

According to [14] and 29], weighting factor $\alpha(\xi)$ can be chosen to be a constant, linear, cubic or trigonometric function in the bridging domain. In the following, we simply choose

$$
\alpha(\xi)=\frac{1}{2}
$$

\section{Adapted energy functions}

In the general case, the internal energy contribution of the continuum is weighted by the factor $\alpha$ and reads

$$
\hat{E}_{c}^{i n t}=\int_{\Omega_{0}^{c}} \alpha(\xi(\mathbf{X})) \Psi(\mathbf{F}) \mathrm{d} V .
$$

In (9), the strain energy density $\Psi=\Psi(\mathbf{F})$ is introduced for infinitesimal volume elements $\mathrm{d} V$ of the body in the material configuration. Any deformation of the body results in a change of the internal energy. Only conservative system are considered here, i.e. there is no dissipation of energy due to the deformation. We, furthermore, concentrate on linear elastic systems at small strains and hence may use symmetric strain tensor

$$
\varepsilon=\frac{1}{2}\left[\frac{\partial \mathbf{u}}{\partial \mathbf{X}}+\left[\frac{\partial \mathbf{u}}{\partial \mathbf{X}}\right]^{T}\right]
$$

wherein $\bullet^{T}$ denotes the transpose. The strain energy density can then be formulated in terms of $\varepsilon$ and material constants $\lambda$ and $\mu$ as follows:

$$
\Psi(\varepsilon)=\frac{\lambda}{2}[\varepsilon: \mathbf{I}]^{2}+\mu\left[\varepsilon^{2}: \mathbf{I}\right] .
$$

The weighted external energy

$$
\hat{E}_{c}^{e x t}=\int_{\partial_{\sigma} \Omega_{0}^{c}} \alpha(\xi(\mathbf{X})) \mathbf{u} \cdot \mathbf{T} \mathrm{d} A+\int_{\Omega_{0}^{c}} \alpha(\xi(\mathbf{X})) \rho_{0} \mathbf{u} \cdot \mathbf{b} \mathrm{d} V
$$

results from body forces $\mathbf{b}$ and surface tractions $\mathbf{T}$ acting on the Neumann boundary $\partial_{\sigma} \Omega_{0}^{c}$ with $\mathrm{d} A$ denoting area elements in the material configuration. In (12), it is assumed that the Neumann boundary $\partial_{\sigma} \Omega_{0}^{c}$ is distinct from the Dirichlet boundary $\partial_{\mathbf{u}} \Omega_{0}^{c}$, i.e.

$$
\partial_{\sigma} \Omega_{0}^{c} \cap \partial_{\mathbf{u}} \Omega_{0}^{c}=\emptyset
$$

The weighted total energy of a static continuum finally follows as

$$
\hat{E}_{c}^{t o t}=\hat{E}_{c}^{i n t}-\hat{E}_{c}^{e x t}
$$

Note that $\alpha(\xi)=1$ holds in the pure continuum, which renders the respective conventional energy formulations of continuum mechanics.

Within the particle domain, the weighted internal energy can be formulated as

$$
\hat{E}_{d}^{i n t}=\hat{E}_{M S}^{i n t}+\hat{E}_{M D}^{i n t}
$$


While $\hat{E}_{d}^{i n t}$ is describing the interactions between the MD particles, the energy of the harmonic bonds between anchor points and MD particles is subsumed in

$$
\hat{E}_{M S}^{i n t}=\frac{1}{2} \sum_{I=1}^{n^{M S}}\left[1-\alpha\left(\xi\left(\mathbf{R}_{I}^{M S}\right)\right)\right] E_{M S I}^{i n t}\left(\mathbf{R}_{I}^{M D}, \mathbf{r}_{I}^{M S}\right),
$$

with $n^{M S}$ being the total number of anchor points. As far as the Arlequin coupling is concerned, it is not necessary to know the exact formulation of $\hat{E}_{M D}^{i n t}$. Since a change of MD particle positions is not allowed during the continuum equilibration, $\hat{E}_{M D}^{i n t}$ remains constant. For sake of completeness, the weighted external energy is given:

$$
\hat{E}_{d}^{e x t}=\hat{E}_{M S}^{e x t}+\hat{E}_{M D}^{e x t}
$$

The weighted external energy of the anchor points can be rewritten as

$$
\hat{E}_{M S}^{e x t}=\sum_{I=1}^{n^{M S}}\left[1-\alpha\left(\xi\left(\mathbf{R}_{I}^{M S}\right)\right)\right] \mathbf{f}_{I}^{A P} \operatorname{ext} \cdot \mathbf{w}_{I},
$$

while the term $\hat{E}_{M D}^{e x t}$ does not change during a continuum equilibration step. Note that the coupled systems discussed in this contribution do not consider external forces acting on anchor points or MD particles. Hence, the total energy of the coupled static system reads:

$$
\hat{E}^{t o t}=\hat{E}_{c}^{i n t}+\hat{E}_{d}^{i n t}-\hat{E}_{c}^{e x t}-\hat{E}_{d}^{e x t}
$$

In case of an uncoupled system, the body can be considered as mechanically equilibrated if its total energy takes a global minimum. However, for coupled systems this approach is not sufficient.

\section{Coupling constraint}

In order to ensure matching of continuum and anchor point displacements in the bridging domain, a coupling constraint is required additionally to the blended energies. Only the anchor points are considered when formulating the coupling constraint, since the remaining MD particles are not visible for the continuum. The match of displacements is realized by evaluating the difference between the continuous displacement field $\mathbf{u}$ and a fictitious displacement field $\mathbf{w}^{*}$ which has to be computed similarly to [14, since there are only discrete displacements available:

$$
\mathbf{w}^{*}=\Pi \mathbf{w} .
$$

In (20), the displacements are defined as

$$
\mathbf{w}=\left\{\mathbf{w}_{1}, \cdots \mathbf{w}_{I}, \cdots \mathbf{w}_{n^{M S}}\right\}^{T}=\mathbf{r}^{M S}-\mathbf{R}^{M S}
$$

with $\mathbf{R}^{M S}$ and $\mathbf{r}^{M S}$ given in terms of position vectors before $\left(\mathbf{R}_{I}^{M S}\right)$ and after $\left(\mathbf{r}_{I}^{M S}\right)$ deformation:

$$
\begin{aligned}
\mathbf{R}^{M S} & =\left\{\mathbf{R}_{1}^{M S}, \cdots \mathbf{R}_{I}^{M S}, \cdots \mathbf{R}_{n^{M S}}^{M S}\right\}^{T} \text { and } \\
\mathbf{r}^{M S} & =\left\{\mathbf{r}_{1}^{M S}, \cdots \mathbf{r}_{I}^{M S}, \cdots \mathbf{r}_{n^{M S}}^{M S}\right\}^{T} .
\end{aligned}
$$

The moving least-square approximation $\Pi$ is discussed in [30]:

$$
\mathbf{w}^{*}(\mathbf{X})=\mathbf{\Phi}(\mathbf{X}) \cdot \mathbf{w}=\sum_{I=1}^{n^{M S}} \Phi_{I}(\mathbf{X})\left[\mathbf{r}_{I}^{M S}-\mathbf{R}_{I}^{M S}\right],
$$

employing the shape function

$$
\mathbf{\Phi}(\mathbf{X})=\left[\Phi_{1}(\mathbf{X}) \ldots \Phi_{n^{M S}}(\mathbf{X})\right]=\mathbf{p}^{T}(\mathbf{X}) \cdot \mathbf{A}^{-1}(\mathbf{X}) \cdot \mathbf{P}^{T} \cdot \mathbf{W}(\mathbf{X})
$$

with the linear basis

$$
\mathbf{p}^{T}=[1, X, Y, Z], \quad \mathbf{X}=[X, Y, Z]^{T},
$$




$$
\mathbf{P}=\left[\begin{array}{c}
\mathbf{p}^{T}\left(\mathbf{R}_{1}^{M S}\right) \\
\vdots \\
\mathbf{p}^{T}\left(\mathbf{R}_{n^{M S}}^{M S}\right)
\end{array}\right],
$$

the moment matrix

$$
\mathbf{A}(\mathbf{X})=\mathbf{P}^{T} \cdot \mathbf{W}(\mathbf{X}) \cdot \mathbf{P}
$$

and the weight function matrix

$$
\mathbf{W}(\mathbf{X})=\left[\begin{array}{cccc}
w_{1}(\mathbf{X}) & 0 & \cdots & 0 \\
0 & w_{2}(\mathbf{X}) & \cdots & 0 \\
\vdots & \vdots & \ddots & \vdots \\
0 & 0 & \cdots & w_{n^{M S}}(\mathbf{X})
\end{array}\right]
$$

The weight function used here is a quartic spline

$$
w_{I}(\mathbf{X})= \begin{cases}1-6 r_{I}^{2}+8 r_{I}^{3}-3 r_{I}^{4} & \text { for } r_{I} \leq 1 \\ 0 & \text { for } r_{I}>1\end{cases}
$$

with

$$
r_{I}=\frac{1}{\rho}\left\|\mathbf{X}-\mathbf{R}_{I}^{M S}\right\|
$$

where $\rho$ is the radius of support.

Hence, the following coupling constraint in terms of $\mathbf{w}^{*}$ is defined to enforce matching displacements:

$$
\left\|\mathbf{u}-\mathbf{w}^{*}\right\|^{2}=\beta_{1} \int_{\Omega^{b}}\left[\mathbf{u}-\mathbf{w}^{*}\right] \cdot\left[\mathbf{u}-\mathbf{w}^{*}\right] \mathrm{d} V+\beta_{2} \int_{\Omega^{b}} \frac{\partial\left[\mathbf{u}-\mathbf{w}^{*}\right]}{\partial \mathbf{X}}: \frac{\partial\left[\mathbf{u}-\mathbf{w}^{*}\right]}{\partial \mathbf{X}} \mathrm{d} V \stackrel{!}{=} 0 .
$$

In case of $\left(\beta_{1}, \beta_{2}\right)=(1,0)$, (31) refers to the $L^{2}$ norm while $\left(\beta_{1}, \beta_{2}\right)=(1,1)$ renders the $H^{1}$ norm. In order to minimize the total energy (19) of the coupled system and to enforce the coupling constraint, the Lagrange multiplier method is applied. Using Lagrange multipliers $\boldsymbol{\lambda}$, the coupling constraint can be rewritten as

$$
b\left(\boldsymbol{\lambda}, \mathbf{u}, \mathbf{r}^{M S}\right)=\beta_{1} \int_{\Omega^{b}} \boldsymbol{\lambda} \cdot\left[\mathbf{u}-\mathbf{w}^{*}\right] \mathrm{d} V+\beta_{2} \int_{\Omega^{b}} \frac{\partial \boldsymbol{\lambda}}{\partial \mathbf{X}}: \frac{\partial\left[\mathbf{u}-\mathbf{w}^{*}\right]}{\partial \mathbf{X}} \mathrm{d} V .
$$

Consequently, the optimization problem can be solved by computing the saddle point of

$$
L\left(\boldsymbol{\lambda}, \mathbf{u}, \mathbf{r}^{M S}\right)=\hat{E}_{t o t}+b\left(\boldsymbol{\lambda}, \mathbf{u}, \mathbf{r}^{M S}\right)
$$

with $L$ being a function of the displacement field $\mathbf{u}$, the Lagrange multiplier field $\boldsymbol{\lambda}$, and the anchor point positions $\mathbf{r}^{M S}$.

\subsubsection{Discretization}

In this section, the discretization of the displacement field $\mathbf{u}$ as well as the Lagrange multiplier field $\boldsymbol{\lambda}$ is discussed in order to solve the optimization problem (33). In both cases, linear basis functions are used:

$$
\begin{aligned}
& \mathbf{u}(\mathbf{X}) \approx \sum_{i=1}^{n_{c}} N_{i}^{c}(\mathbf{X}) \mathbf{u}_{i}^{h}=\mathbf{N}^{c}(\mathbf{X}) \cdot \mathbf{u}^{h} \\
& \boldsymbol{\lambda}(\mathbf{X}) \approx \sum_{j=1}^{n_{\lambda}} N_{j}^{\lambda}(\mathbf{X}) \boldsymbol{\lambda}_{j}^{h}=\mathbf{N}^{\lambda}(\mathbf{X}) \cdot \boldsymbol{\lambda}^{h}
\end{aligned}
$$

$\mathbf{u}_{i}^{h}$ and $\boldsymbol{\lambda}_{j}^{h}$ denote the nodal values, $N_{i}^{c}$ and $N_{j}^{\lambda}$ are the respective basis functions, while $n_{c}$ and $n_{\lambda}$ describe the number of nodes in the discretized continuum and the Lagrange multiplier space. The nodal values are assembled into the vectors $\mathbf{u}^{h}$ and $\boldsymbol{\lambda}^{h}$. Hence, $L$ becomes a function of the discretized displacements and Lagrange multipliers:

$$
L\left(\boldsymbol{\lambda}, \mathbf{u}, \mathbf{r}^{M S}\right) \approx L\left(\boldsymbol{\lambda}^{h}, \mathbf{u}^{h}, \mathbf{r}^{M S}\right) .
$$


The set of variables can be summarized in a vector $\mathbf{d}=\left[\boldsymbol{\lambda}^{h}, \mathbf{u}^{h}, \mathbf{r}^{M S}\right]^{T}$ which allows to define the necessary condition for a saddle point of (33):

$$
\delta_{\mathbf{d}} L(\mathbf{d})=\delta_{\mathbf{d}} \hat{E}_{t o t}\left(\mathbf{u}^{h}, \mathbf{r}^{M S}\right)+\delta_{\mathbf{d}} b(\mathbf{d})=0 .
$$

The resulting set of equations can be structured into three parts:

\section{FE domain}

The relations in the FE domain are obtained from the variation of $L$ with respect to the nodal displacements $\mathbf{u}^{h}$ :

$$
\delta_{\mathbf{u}^{h}} L=0 \longrightarrow\left\{\begin{array}{lll}
\hat{\mathbf{f}}_{c}^{i n t}\left(\mathbf{u}^{h}\right) & \hat{\mathbf{f}}_{c}^{\text {ext }} \\
\mathbf{f}_{b}^{i n t}\left(\mathbf{u}^{h}\right)+\mathbf{f}^{c p l}\left(\boldsymbol{\lambda}^{h}\right) & = & \mathbf{f}_{b}^{e x t}
\end{array}\right\}
$$

The internal forces in the pure FE domain and in the bridging domain are given by $\hat{\mathbf{f}}_{c}^{\text {int }}$ and $\mathbf{f}_{b}^{\text {int }}$, while $\mathbf{f}^{c p l}$ denotes the vector of coupling forces due to the coupling constraint. The external force vectors in the respective domains are represented by $\hat{\mathbf{f}}_{c}^{e x t}$ and $\mathbf{f}_{b}^{e x t}$. In case of a linear stress-strain relation in the continuum, the internal force vectors can be written as

$$
\begin{aligned}
{\left[\begin{array}{l}
\hat{\mathbf{f}}_{c}^{i n t} \\
\mathbf{f}_{b}^{\text {int }}
\end{array}\right] } & =\mathbf{K}^{c} \cdot\left[\begin{array}{l}
\hat{\mathbf{u}}^{c} \\
\mathbf{u}^{b}
\end{array}\right]=\mathbf{K}^{c} \cdot \mathbf{u}^{h} \text { and } \\
\mathbf{f}^{c p l} & =\mathbf{G}_{c \lambda} \cdot \boldsymbol{\lambda}^{h} .
\end{aligned}
$$

Here, a $\left(n_{c}^{\text {dof }} \times n_{c}^{\text {dof }}\right)$ stiffness matrix $\mathbf{K}^{c}$ in the continuum and a $\left(n_{c b}^{\text {dof }} \times n_{\lambda}^{\text {dof }}\right)$ coupling matrix $\mathbf{G}_{c \lambda}$ in the bridging domain is used. $n_{c}^{d o f}$ is the number of FE degrees of freedom in the continuum $\Omega^{c}, n_{c b}^{d o f}$ the number of FE degrees of freedom in the bridging domain $\Omega^{b}$, and $n_{\lambda}^{\text {dof }}$ the number of degrees of freedom of the Lagrange multipliers. Furthermore, the vector of nodal displacements $\mathbf{u}^{h}$ is separated into a vector of nodal displacements in the pure $\mathrm{FE}$ domain $\hat{\mathbf{u}}^{c}$ and a vector of displacements in the bridging domain $\mathbf{u}^{b}$.

\section{Particle domain}

The system of equations for the particle domain follows as:

$$
\delta_{\mathbf{w}} L=0 \longrightarrow\left\{-\mathbf{f}^{M S}(\mathbf{w})+\mathbf{f}^{A P c p l}\left(\boldsymbol{\lambda}^{h}\right)-\mathbf{f}^{A P}=\mathbf{f}^{A P e x t}\right\}
$$

In (41), the vectors $\mathbf{f}^{M S}$ and $\mathbf{f}^{A P}$ subsume all anchor point forces as introduced in (3), multiplied by $[1-\alpha]$, while $\mathbf{f}^{A P c p l}$ is the vector of all forces that are exerted on anchor points due to the coupling constraint. For sake of completeness, the vector of external forces $\mathbf{f}^{A P}$ ext acting on anchor points is given as well:

$$
\begin{gathered}
I \text {-th component of } \mathbf{f}^{A P}(\text { force vector of anchor point } I):\left[1-\alpha\left(\xi\left(\mathbf{R}_{I}^{M S}\right)\right)\right] \mathbf{f}_{I}^{A P}, \\
I \text {-th component of } \mathbf{f}^{A P \text { ext }}(\text { force vector of anchor point } I):\left[1-\alpha\left(\xi\left(\mathbf{R}_{I}^{M S}\right)\right)\right] \mathbf{f}_{I}^{A P} \text { ext }
\end{gathered}
$$

Since only harmonic interaction potentials between anchor points and superatoms are considered in the present formalism, a $\left(n_{d}^{d o f} \times n_{d}^{d o f}\right)$ stiffness matrix $\mathbf{K}^{d}$ can be introduced with $n_{d}^{d o f}=3 n^{M S}$. Based on (41) it reads

$$
\mathbf{K}^{d}=\left[\begin{array}{ccc}
\mathbf{K}_{\alpha 1}^{A P} & 0 & 0 \\
0 & \ddots & 0 \\
0 & 0 & \mathbf{K}_{\alpha n^{M S}}^{A P}
\end{array}\right] ; \quad \mathbf{K}_{\alpha I}^{A P}=\left[1-\alpha\left(\xi\left(\mathbf{R}_{I}^{M S}\right)\right)\right] \mathbf{K}_{I}^{A P} .
$$

Furthermore, a $\left(n_{d}^{d o f} \times n_{\lambda}^{d o f}\right)$ coupling matrix $\mathbf{G}_{d \lambda}$ is used as follows:

$$
\mathbf{f}^{A P c p l}=\mathbf{G}_{d \lambda} \cdot \lambda^{h}
$$




\section{Lagrange multipliers}

The variation of $L$ with respect to the Lagrange multipliers leads to:

$$
\begin{gathered}
\delta_{\boldsymbol{\lambda}^{h}} L=0 \longrightarrow\left\{\mathbf{G}_{\lambda c} \cdot \mathbf{u}^{b}+\mathbf{G}_{\lambda d} \cdot \mathbf{w}=\mathbf{0}\right\} \quad \text { with } \\
\mathbf{G}_{\lambda c}=\mathbf{G}_{c \lambda}^{T} \text { and } \mathbf{G}_{\lambda d}=\mathbf{G}_{d \lambda}^{T}
\end{gathered}
$$

Thus, the complete system of equations can be formulated as follows:

$$
\delta_{\mathbf{u}^{h}, \mathbf{w}, \boldsymbol{\lambda}^{h}} L=0 \longrightarrow\left\{\begin{array}{lll}
\hat{\mathbf{f}}_{c}^{i n t}\left(\mathbf{u}^{h}\right) & \hat{\mathbf{f}}_{c}^{\text {ext }} \\
\mathbf{f}_{b}^{\text {int }}\left(\mathbf{u}^{h}\right)+\mathbf{G}_{c \lambda} \cdot \boldsymbol{\lambda}^{h} & = & \mathbf{f}_{b}^{\text {ext }} \\
\mathbf{K}^{d} \cdot \mathbf{w}+\mathbf{G}_{d \lambda} \cdot \boldsymbol{\lambda}^{h} & = & \mathbf{f}^{A P e x t}+\mathbf{f}^{A P} \\
\mathbf{G}_{\lambda c} \cdot \mathbf{u}^{b}+\mathbf{G}_{\lambda d} \cdot \mathbf{w}= & \mathbf{0}
\end{array}\right\}
$$

In case of a linear elastic continuum and in absence of external forces acting on anchor points, the system of equations becomes linear and can be written as:

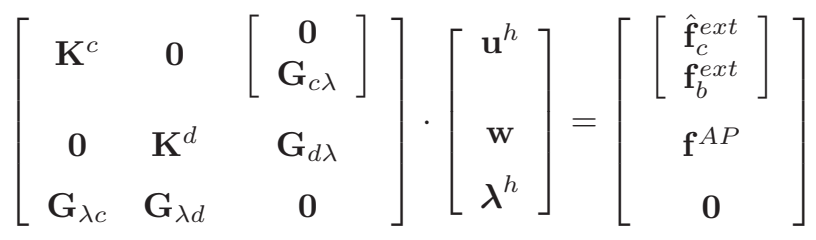

Concluding this section, (47) describes the coupling between the finite element domain and the anchor points, treating the MD region as a domain that interacts with its surrounding only via anchor points. Important quantities are, among others, the stiffness of the finite element domain and of the anchor points, represented by the stiffness matrices $\mathbf{K}^{c}$ and $\mathbf{K}^{d}$, the coupling forces $\mathbf{f}^{c p l}$ and $\mathbf{f}^{A P c p l}$, expressed in terms of the Lagrange multipliers $\lambda^{h}$ as well as the coupling matrices $\mathbf{G}_{c \lambda}$ and $\mathbf{G}_{d \lambda}$, the forces exterted on anchor points by the MD domain $\mathbf{f}^{A P}$, and, of course, the nodal displacements $\mathbf{u}^{h}$ and the displacements of anchor points $\mathbf{w}^{h}$. In the following section, we will focus on the implementation of the relations developed so far and discuss some important technical aspects.

\section{Coupling Scheme}

\subsection{Staggered coupling of FE and MD}

Based on the definitions and equations introduced in the previous sections, the staggered coupling procedure is described in the following. As stated before, an iterative solution scheme is mandatory to solve the basic equations for the coupled system. On the one hand, the positions of anchor points change due to the deformation of the continuum while the superatoms of the MD domain remain fixed (coupling between continuum and anchor points). On the other hand, the MD particles move within a frame of fixed anchor points (anchor points tethered to MD system), cf. Figure 5, until the reaction forces on the anchor points are converged within their statistical-mechanical variation. This procedure has to be continued until the deformed coupled system is in equilibrium.

This contribution focusses on MD systems at small deformations, i.e. showing a nearly linear stressstrain relation. Thus, it is possible to consider a linear elastic FE system that can be computed by solving the system (47) of linear equations. The staggered algorithm is set up as follows:

\section{1. initial MD-FE iteration step: $n=1$}

(a) initial MD run: the MD system is set up, the anchor points are defined, the system is equilibrated, the reaction forces are sampled; output data:

- $\mathbf{r}_{(1)}^{M D}$ : current positions of superatoms in the first MD-FE iteration step $n=1$

- $\mathbf{R}_{(1)}^{M S}$ : initial anchor point positions in the first MD-FE iteration step $n=1$ (chosen according to 22$]$ )

- $\left\langle\hat{\mathbf{f}}^{A P}\right\rangle_{\mathcal{T}(1)}$ : time average of forces exerted on anchor points by superatoms, i.e. by the MD domain ("reaction forces") 
(a)

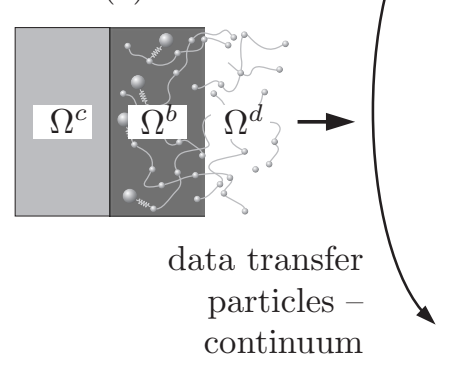

continuum coupled to anchor points

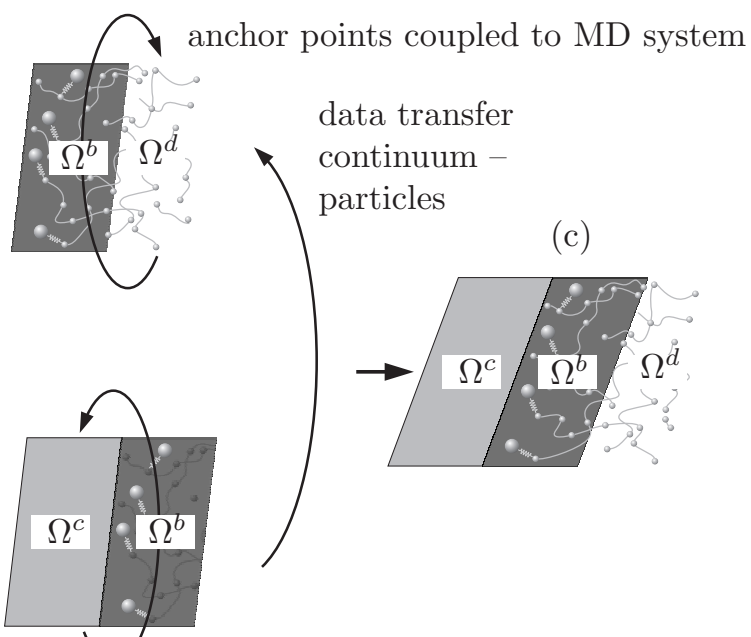

Figure 5: Concept of the staggered coupling scheme: initial configuration (a), coupling procedure (b): equilibration of continuum coupled to anchor points (bottom, merely the anchor points are "visible" to the continuum, the MD particles are fixed), data transfer (anchor point displacements) to the particle system, equilibration of the particle system (top, fixed anchor points represent the continuum enclosing the particles), data transfer (forces exerted by the particles on the anchor points) back to the continuum, deformed and equilibrated system (c)

(b) initial FE equilibration run: the FE system is set up, the bridging domain is defined such that it contains all anchor points; the FE system is deformed due to Dirichlet and Neumann boundary conditions; thus, anchor points are moved and form a new boundary for the MD system;

input data:

- $\mathbf{X}^{F E}, \mathbf{R}_{(1)}^{M S}$ : initial positions of FE nodes and anchor points

- $\mathbf{f}_{(1)}^{A P}=\left\langle\hat{\mathbf{f}}^{A P}\right\rangle_{\mathcal{T}(1)}$ : the time average of forces exerted on anchor points is interpreted as a static section force vector between anchor points and their associated superatoms output data (computed by solving (46) or (47)):

- $\mathbf{x}_{(1)}^{F E}$ : current positions of FE nodes after the first FE-MD iteration step

- $\mathbf{r}_{(1)}^{M S}$ : current anchor point positions after the first FE-MD iteration step

\section{2. i-th MD-FE iteration step: $n=i$}

(a) i-th MD run: MD equilibration according to updated anchor point positions followed by sampling of the reaction forces input data:

- $\mathbf{R}_{(i)}^{M D}=\mathbf{r}_{(i-1)}^{M D}$ : initial positions of superatoms in the $i$-th MD-FE iteration step

- $\mathbf{R}_{(i)}^{M S}=\mathbf{r}_{(i-1)}^{M S}$ : initial anchor point positions in the $i$-th MD-FE iteration step output data:

- $\mathbf{r}_{(i)}^{M D}$ : current positions of superatoms in the $i$-th MD-FE iteration step

- $\left\langle\hat{\mathbf{f}}^{A P}\right\rangle_{\mathcal{T}(i)}$

(b) i-th FE equilibration run: due to updated anchor point forces, the FE system is equilibrated; input data:

- $\mathbf{X}^{F E}$ : initial positions of FE nodes (independent of MD-FE iteration step)

- $\mathbf{R}_{(i)}^{M S}$ : initial anchor point positions in the $i$-th MD-FE iteration step

- $\mathbf{f}_{(i)}^{A P}=\left\langle\hat{\mathbf{f}}^{A P}\right\rangle_{\mathcal{T}(i)}$ 
output data (computed by solving (46) or (47)):

- $\mathbf{x}_{(i)}^{F E}$ : current positions of FE nodes after the $i$-th FE-MD iteration step

- $\mathbf{r}_{(i)}^{M S}$ : current anchor point positions after the $i$-th FE-MD iteration step

3. continue with subsequent MD-FE iteration steps until a criterion for convergence is met;

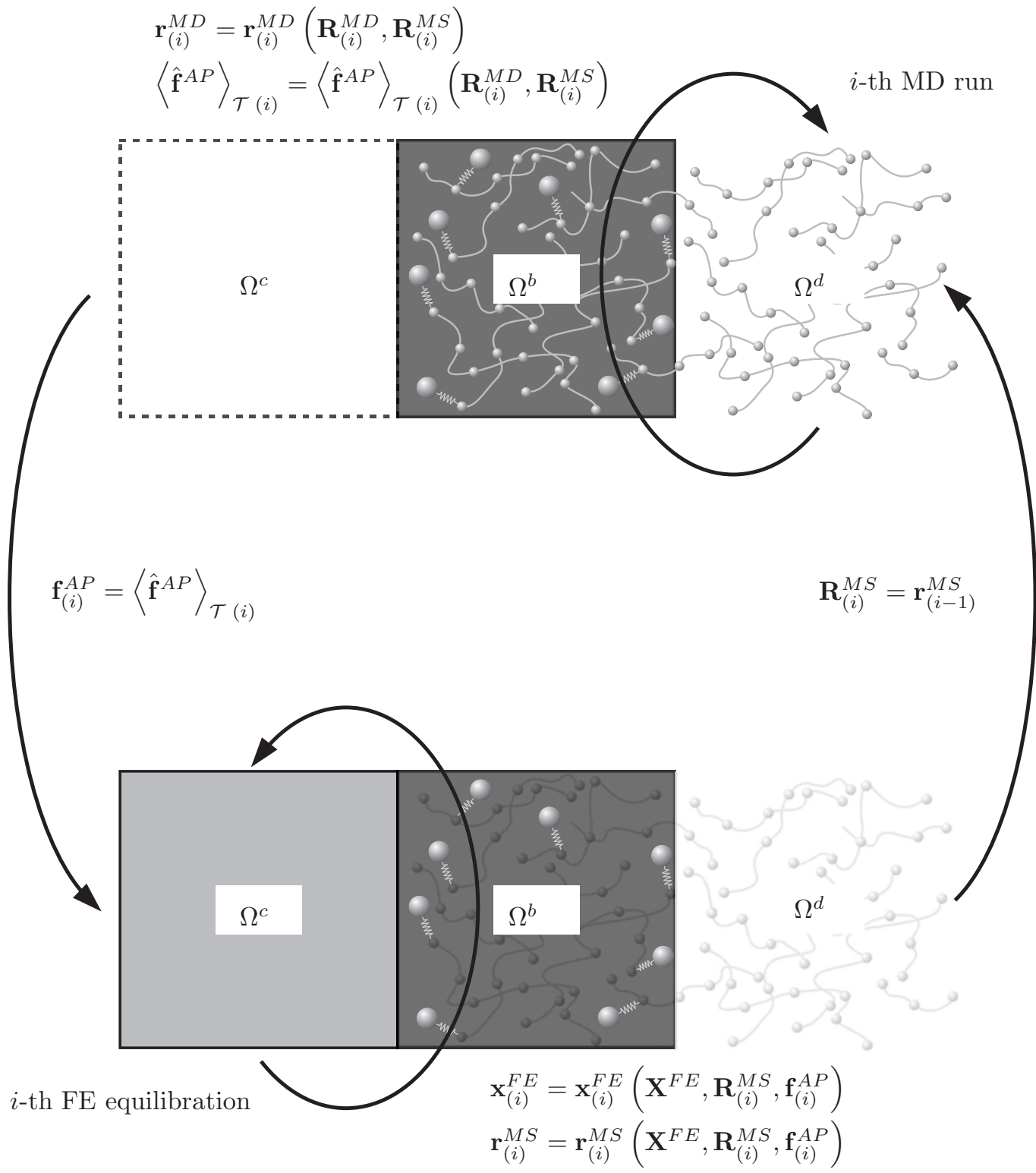

Figure 6: $i$-th MD-FE iteration step combines the $i$-th MD run (top) and the $i$-th FE equilibration (bottom): during the MD run an update of MD particle positions $\mathbf{r}_{(i)}^{M D}$ and computation of the time average of forces acting on anchor points $\left\langle\hat{\mathbf{f}}^{A P}\right\rangle_{\mathcal{T}(i)}$ is performed, the FE equilibration run delivers updated positions of FE nodes and anchor points; data is transferred from MD to FE and vice versa

This procedure is visualized in Figure 6 the $i$-th MD-FE iteration step is defined by an MD run that provides updated positions of superatoms $\mathbf{r}_{(i)}^{M D}$ and the time average $\left\langle\hat{\mathbf{f}}^{A P}\right\rangle_{\mathcal{T}(i)}$ of forces exerted on the anchor points by the superatoms. Both quantities are computed as a function of the initial positions of superatoms $\mathbf{R}_{(i)}^{M D}$ and anchor points $\mathbf{R}_{(i)}^{M S}$. During this MD run, the FE domain is not "visible" to the MD domain, since the influence of the FE domain is transferred into the MD region only by the 
anchor points. The time average $\left\langle\hat{\mathbf{f}}^{A P}\right\rangle_{\mathcal{T}(i)}$ is used as input for the subsequent FE equilibration such that $\mathbf{f}_{(i)}^{A P}=\left\langle\hat{\mathbf{f}}^{A P}\right\rangle_{\mathcal{T}(i)}$. In this step, the current positions of FE nodes $\mathbf{x}_{(i)}^{F E}$ as well as the positions of anchor points $\mathbf{r}_{(i)}^{M S}$ are determined as a function of the initial nodal positions $\mathbf{X}^{F E}$, the initial anchor point positions $\mathbf{R}_{(i)}^{M S}$ of the current FE-MD iteration step $i$, and $\mathbf{f}_{(i)}^{A P}$. Reciprocally to the MD run, now the particle domain is not "visible". Only the anchor points represent the MD system during the FE computation. In the next MD-FE iteration step, the updated anchor point positions serve as input for the MD computation: $\mathbf{R}_{(i)}^{M S}=\mathbf{r}_{(i-1)}^{M S}$. This procedure is carried out until a criterion for convergence is met, which will be discussed later.

\section{Remark on the displacements of anchor points}

In order to apply (47) to a linear elastic FE system at an arbitrary MD-FE iteration step $i$, some adaptions are required: the third set of equations of (47), which essentially represents the geometric coupling constraint, is formulated with nodal displacements $\mathbf{u}^{h}=\mathbf{x}^{F E}-\mathbf{X}^{F E}$, representing the difference between current positions and the intial FE configuration.

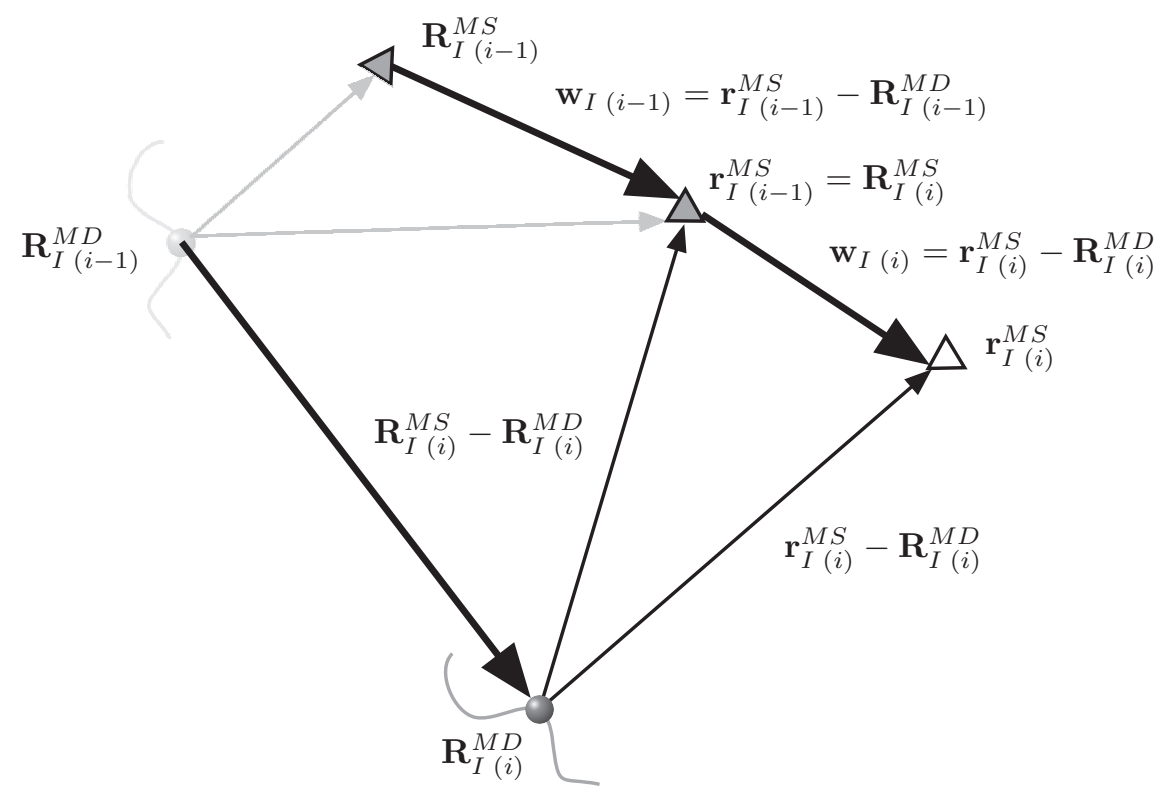

Figure 7: Positions of anchor point $I$ and its associated superatom in the $(i-1)$-th (top) and $i$-th (bottom) MD-FE iteration step

Thus, at the $i$-th MD-FE iteration it reads

$$
\mathbf{u}_{(i)}^{h}=\mathbf{x}_{(i)}^{F E}-\mathbf{X}^{F E} .
$$

To fulfill the coupling constraint properly, the total displacement of anchor points with respect to their original positions $\mathbf{R}_{(1)}^{M S}$ at the first iteration step has to be considered:

$$
\mathbf{w}_{(i)}^{t o t}=\mathbf{r}_{(i)}^{M S}-\mathbf{R}_{(1)}^{M S}
$$

However, the energy of the particle region that is "visible" in the FE domain is restricted to the energy of bonds between anchor points and their associated superatoms, i.e. at the $i$-th MD-FE iteration step it reads

$$
E_{M S I(i)}^{i n t}=\frac{1}{2} k_{I}\left|\mathbf{r}_{I(i)}^{M S}-\mathbf{R}_{I(i)}^{M D}\right|^{2} \neq \frac{1}{2} k_{I}\left|\mathbf{w}_{I(i)}^{t o t}\right|^{2} .
$$

Taking the derivative of (50) with respect to the position of the $I$-th anchor point in the $i$-th iteration 
step, it follows according to Figure 7 .

$$
\begin{aligned}
\mathbf{f}_{I(i)}^{t o t} & =-\frac{\partial E_{M S I(i)}^{i n t}}{\partial \mathbf{r}_{I(i)}^{M S}} \\
& =-k_{I}\left[\mathbf{r}_{I(i)}^{M S}-\mathbf{R}_{I(i)}^{M D}\right]=-k_{I}\left[\mathbf{r}_{I(i)}^{M S}-\mathbf{R}_{I(i)}^{M S}\right]-k_{I}\left[\mathbf{R}_{I(i)}^{M S}-\mathbf{R}_{I(i)}^{M D}\right] \\
& =-k_{I} \mathbf{w}_{I(i)}+\mathbf{f}_{I(i)}^{A P}=-k_{I} \mathbf{w}_{I(i)}-k_{I} \sum_{j=1}^{i-1} \mathbf{w}_{I(j)}+k_{I} \sum_{j=1}^{i-1} \mathbf{w}_{I(j)}+\mathbf{f}_{I(i)}^{A P} \\
& =-k_{I} \sum_{j=1}^{i} \mathbf{w}_{I(j)}+k_{I}\left[\mathbf{r}_{I(i-1)}^{M S}-\mathbf{R}_{I(1)}^{M S}\right]+\mathbf{f}_{I(i)}^{A P} \\
& =-k_{I} \mathbf{w}_{I(i)}^{t o t}+k_{I}\left[\mathbf{R}_{I(i)}^{M S}-\mathbf{R}_{I(1)}^{M S}\right]+\mathbf{f}_{I(i)}^{A P}
\end{aligned}
$$

With weighting factor $\alpha(\xi)$ this can be rewritten as

$$
\mathbf{f}_{(i)}^{t o t}=-\frac{\partial E_{M S(i)}^{i n t}}{\partial \mathbf{r}_{(i)}^{M S}}=-\mathbf{K}^{d} \cdot \mathbf{w}_{(i)}^{t o t}+\mathbf{K}^{d} \cdot\left[\mathbf{R}_{(i)}^{M S}-\mathbf{R}_{(1)}^{M S}\right]+\mathbf{f}_{(i)}^{A P}
$$

with

$$
\begin{array}{cc}
I \text {-th component of } \mathbf{f}_{(i)}^{A P}: & {\left[1-\alpha\left(\xi\left(\mathbf{R}_{I}^{M S}\right)\right)\right] \mathbf{f}_{I(i)}^{A P},} \\
I \text {-th component of } \mathbf{f}_{(i)}^{t o t}: & {\left[1-\alpha\left(\xi\left(\mathbf{R}_{I}^{M S}\right)\right)\right] \mathbf{f}_{I(i)}^{t o t} .}
\end{array}
$$

Thus, (47) can be reformulated for the $i$-th MD-FE iteration step:

$$
\left[\begin{array}{ccc}
\mathbf{K}^{c} & \mathbf{0} & {\left[\begin{array}{c}
\mathbf{0} \\
\mathbf{G}_{c \lambda}
\end{array}\right]} \\
\mathbf{0} & \mathbf{K}^{d} & \mathbf{G}_{d \lambda} \\
\mathbf{G}_{\lambda c} & \mathbf{G}_{\lambda d} & \mathbf{0}
\end{array}\right] \cdot\left[\begin{array}{c}
\mathbf{u}_{(i)}^{h} \\
\mathbf{w}_{(i)}^{t o t} \\
\boldsymbol{\lambda}_{(i)}^{h}
\end{array}\right]=\left[\begin{array}{l}
{\left[\begin{array}{l}
\hat{\mathbf{f}}_{c}^{e x t} \\
\mathbf{f}_{b}^{e x t}
\end{array}\right]} \\
\mathbf{f}_{(i)}^{A P}+\mathbf{K}^{d} \cdot\left[\mathbf{R}_{(i)}^{M S}-\mathbf{R}_{(1)}^{M S}\right] \\
\mathbf{0}
\end{array}\right]
$$

\subsection{Initialization of a coupled MD-FE simulation}

In order to carry out a coupled MD-FE computation, some technical aspects have to be discussed. Before the coupling procedure can be applied as described in the previous section, the system has to be set up: the continuum has to be defined and a FE mesh must be created. Secondly, the particle domain must be prepared in a way that it can replace a part of the FE domain. Furthermore, the match between the different regions has to be ensured by defining the bridging domain. The systems investigated in the following consist of a hollow cube discretized by finite elements and a cubic particle domain that is fully embedded into the FE domain, cf. Figure 8.

1. The cubic particle domain is set up by defining the box size of the MD system, and, among others, the kind of the particles, their interactions, temperature, pressure, etc. This system is equilibrated using standard periodic boundary conditions without defining any anchor points. This first equilibration procedure generates the initial configuration of the particle domain, which is cooled down below the glass transition temperature. Furthermore, the elastic constants (Young's modulus and Poisson's ratio) of the polymer can be obtained from uniaxial MD-tension tests (parameter identification).

2. The particles (or superatoms in case of coarse grained simulations as carried out here) tethered to anchor points are selected using a probability function in the boundary domain. Without going into detail here, this probability function can be chosen as constant, linear, or as an exponential decay function. After that, the corresponding anchor points are created. The particle domain is now surrounded by stochastic boundary conditions and ready to be coupled to the FE domain. 


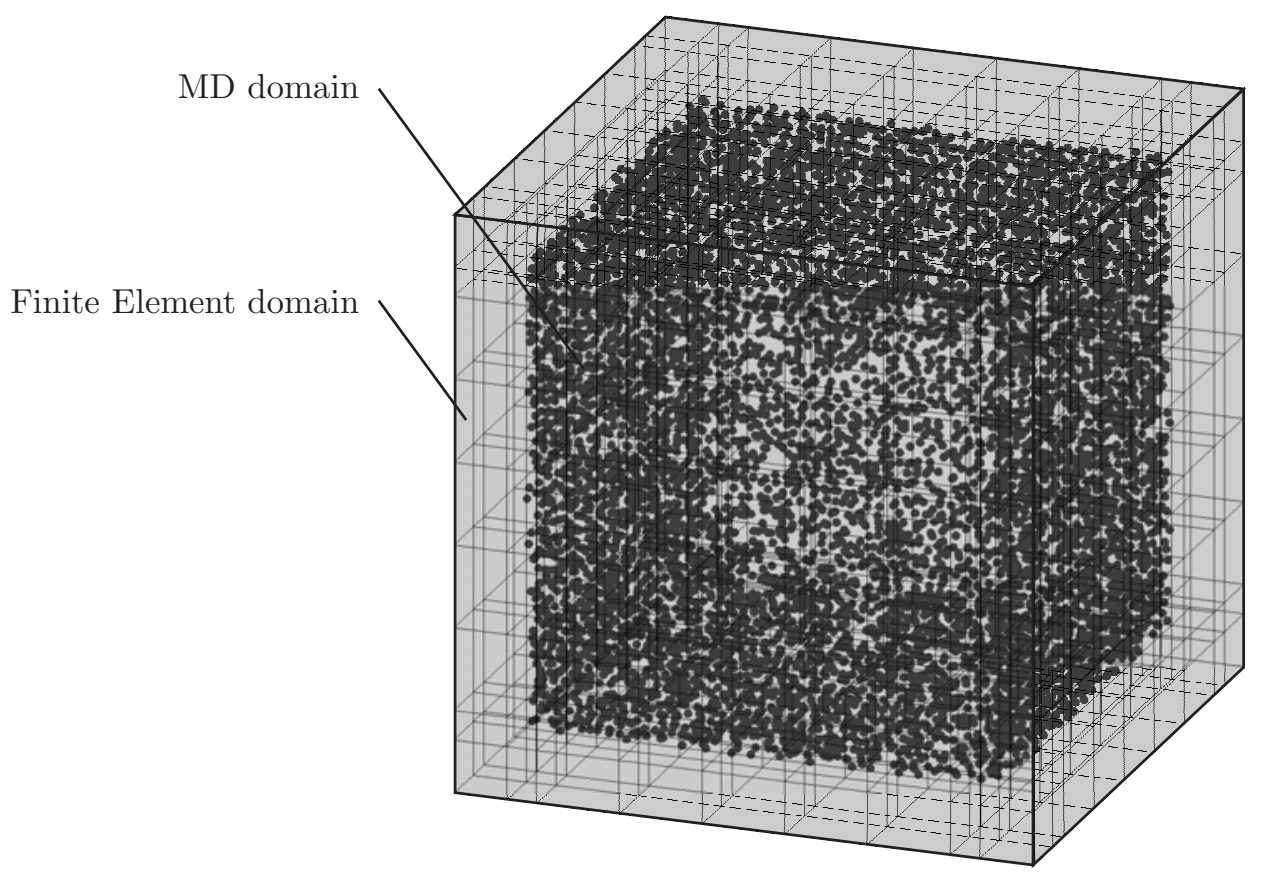

Figure 8: Considered simulation box: hollow FE cube filled with MD system

3. Within step 2, the initial anchor point positions have been distributed randomly. Thus one cannot expect to start the coupled simulation from an equilibrated configuration. As a matter of fact some initial simulation steps are required in order to relax the MD system under SBC. Numerical tests have shown that this initial relaxation significantly reduces the number of iterations required for the subsequent coupled deformation simulations. Furthermore, the same initial MD configuration can then be re-used with different boundary conditions. Because the anchor points cannot be moved in a pure MD simulation, the coupling algorithm itself is used to set up the equilibrated initial particle system. Thereby, a rather weak (low stiffness) FE domain, which mimics free space without matter, is employed during this initial relaxation. From a theoretical point of view, the stiffness would ideally be set to zero (i.e. the FE domain would simply not exist) which, of course, is numerically unfeasible.

4. The coupled MD-FE simulation is carried out. Instead of the previous one, a new FE mesh is defined matching the relaxed configuration of the MD domain as obtained in step 3 Realistic elastic constants as provided by the parameter identification of the pure MD system (step 10) are used from now on.

\section{Numerical examples}

In this section, coupled MD-FE simulations are benchmarked against pure FE simulations and some criteria suitable to demonstrate the capability of the new hybrid method are introduced. When considering homogeneous materials at small strains, similar results should be obtained in case of coupled simulations and pure FE computations.

\subsection{System configuration}

Atactic polystyrene (PS) in CG representation (1 CG bead per chemical repeat unit) [19] is employed to demonstrate the coupling method. The CG potentials, derived by iterative Boltzmann inversion at $590 \mathrm{~K}$ and $101.3 \mathrm{kPa}$, have been optimized by minimizing the difference between radial distribution functions of a chosen atomistic reference system and the CG profile. The PS sample is represented by 300 polymer chains, each consisting of 200 CG beads. The initial configurations are generated as self-avoiding random walks (SARW) at low density $\left(0.7 \mathrm{kgm}^{-3}\right)$ in order to avoid overlap of the CG beads. Under conventional PBCs, the equilibration of the polymer required up to $20 \mathrm{~ns}$, using a time 
step $\Delta t=5 \mathrm{fs}$. In order to prepare the particle system for the coupling procedure, the temperature is reduced to $T=100 \mathrm{~K}$, which is well below the glass transition temperature of PS in CG model $\left(T_{g}=170 \mathrm{~K},[31]\right)$. The constant cooling rate is $10 \mathrm{Kns}^{-1}$, the equilibrated box size is $20.8 \mathrm{~nm}$ in each direction.

After that, the boundary conditions are switched from periodic to nonperiodic, using the final PBC configuration as the initial state for the subsequent SBC simulation. Polymer chains, which are not located completely in the final PBC box, are divided into smaller fragments. The presence of shortened polymer chains may locally lead to differences in the material properties, which will be discussed later. The thickness of the effective boundary region is chosen as $1.5 \mathrm{~nm}$, which equals the cutoff radius of the particle simulation, whereas the size of the DPD region is $0.5 \mathrm{~nm}$. Using the same overall box size as in case of $\mathrm{PBC}$, the edge length of the central MD region consequently follows as $16.8 \mathrm{~nm}$.

In the next step, the anchor points are defined. In order to prevent a loss of MD particles from the simulation box, an exponential decay of the anchor point density is chosen: the density is large at the interface between bridging and pure FE domain and decreases to zero at the interface to the $\mathrm{DPD}$ region. From the MD point of view, the boundary region is surrounded by a vacuum since the continuum is not "visible" during the MD run. However, the stochastic dynamics and the anchor points as part thereof pretend a large number of particles outside the simulation box. In our approach, these "pseudo" particles outside the MD box represent the continuum. As always in MD simulations without periodic boundary conditions, the particles have a certain chance to leave the particle domain and to enter the vacuum. The physical meaning of the anchor points thus is twofold. They act as information transmitter between continuum and particle domain and hinder the MD particles from disappearing into the vacuum. The influence of the anchor point concentration and magnitude of the force constant between anchor point and MD particle on the number of transitions of MD particles into the vacuum has been studied in detail in one of our recent articles 22 .

The final simulation box contains 300 polymer chains, each consisting of 200 superatoms, which in total amounts to 60,000 superatoms, with approximately 29,000 of these being located in the boundary region. Furthermore, 9004 anchor points are defined and coupled to MD particles using a force constant $k_{I}=1400 \mathrm{kJmol}^{-1} \mathrm{~nm}^{-2}=2.32 \mathrm{Nm}^{-1}$. This value is of the same order of magnitude as employed between bonded CG beads in the MD region, a choice which prevents larger system perturbations and which allows to keep the timestep at 5 fs. Furthermore, the DPD equations of motion are solved with a friction coefficient of $10 \mathrm{pNpsnm}^{-1}$. With this setup an efficient temperature control is possible $(T=99.85 \pm 0.4 \mathrm{~K})$. Before coupling the particle system to the FE domain an additional equilibration of $5 \mathrm{~ns}$ is performed.
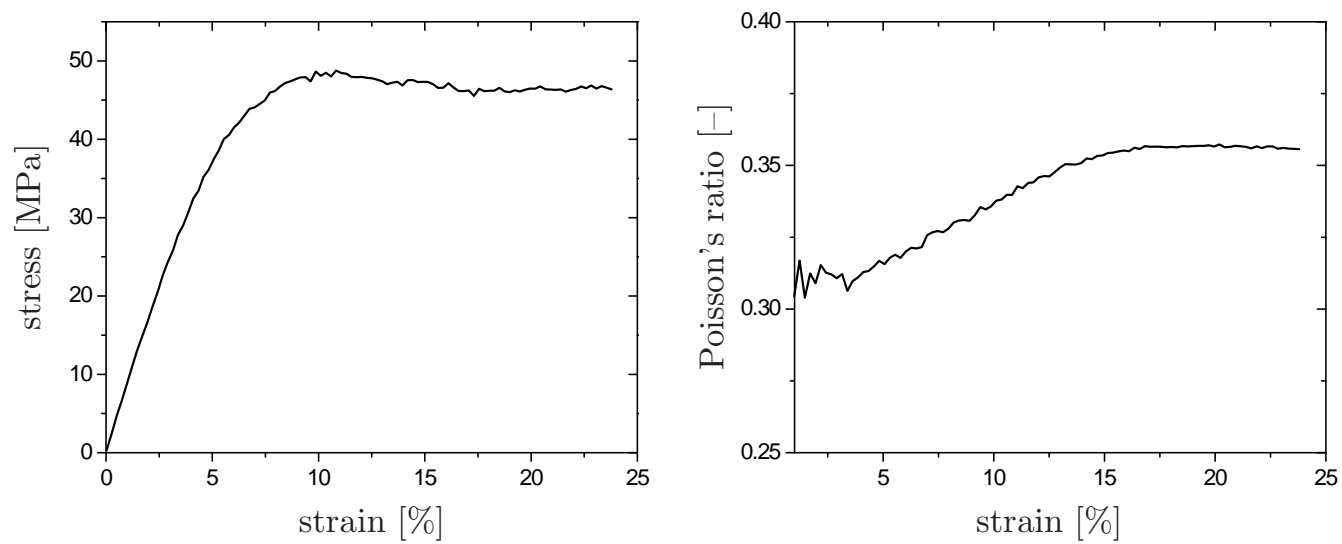

Figure 9: Parameter identification from the pure particle system: stress-strain curve (left) and Poisson's ratio as a function of strain (right). Both diagrams have been obtained from a uniaxial tension test based on a coarse grained simulation of polystyrene at $100 \mathrm{~K}, T_{g}=170 \mathrm{~K}$, the deformation rate is 10 $\mathrm{nm} / \mathrm{ns}$, the stress is computed with respect to the deformed geometry, from 31 .

The material parameters to be used in the FE domain are identified from a pure MD simulation under PBCs. Recently we have studied the mechanical behaviour of PS in CG resolution under uniaxial deformation [31. Figure 9] shows the stress-strain curve of PS at $100 \mathrm{~K}$ obtained from a numerical uniaxial tension test, employing a deformation rate of $10 \mathrm{~nm} / \mathrm{ns}$. Up to $3 \%$ strain, the stress-strain relation is approximately linear, after that it becomes clearly nonlinear and around $7 \%$ strain, the yield 
point can be observed. A Young's modulus of $\left.E\right|_{100 \mathrm{~K}}=827 \mathrm{MPa}$ is computed from the slope of the linear part of the stress-strain curve. Furthermore, Poisson's ratio is slightly higher than 0.3 , which is close to the value identified from atomistic simulations 32 . It has to be mentioned that, due to the CG description, the Young's modulus is smaller than in atomistic simulations or experiments. The reasons for that, together with an appropriate scaling procedure, are discussed in detail in 31. Additionally, it is worth noting that the FE domain is treated isothermally, i.e. no heat conduction is simulated. The material parameters $\left.E \equiv E\right|_{100 \mathrm{~K}}$ and $\left.\nu \equiv \nu\right|_{100 \mathrm{~K}}$ hence represent a continuum at the same temperature as prescribed to the MD domain, i.e. at $100 \mathrm{~K}$. In case of specifying a different temperature in the particle region, additional parameter identifications would be required.

With the linear elastic material parameters at hand, the FE domain is set up according to the size of the particle domain. In this contribution, a hollow cube is chosen with an edge length of $30 \mathrm{~nm}$, whereas the bridging domain $\Omega^{b}$ coincides with the effective boundary region of the particle system. The system is deformed symmetrically by prescribing displacements in $y$-direction at the upper and lower $x z$-surfaces, cf. Figure 10
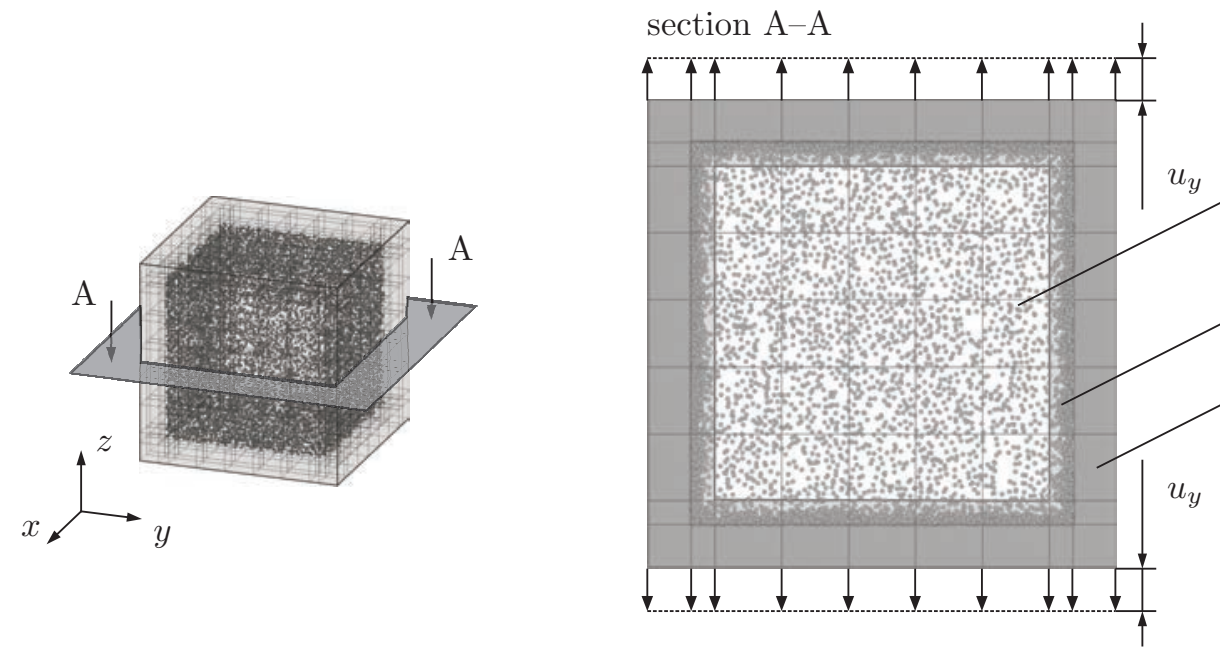

pure MD domain $\Omega^{d}$ (only anchor points at the boundaries of the bridging domain are visible)

\section{bridging domain $\Omega^{b}$}

pure FE domain $\Omega^{c}$

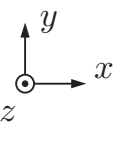

Figure 10: Coupled system subjected to prescribed displacements $u_{y}$ at the top and bottom $x z$-surfaces, $3 \mathrm{~d}$ view (left) and sectional view $\mathrm{A}-\mathrm{A}$ (right)

However, before applying any deformation, an initial equilibration is required in order to find an appropriate starting configuration of the MD particles and anchor points, please see also Section 3.2. step 3. This is necessary due to the following reasons: on the one hand, the anchor point positions are not in equilibrium since they have been chosen randomly and on the other hand, shorter chain lengths caused by switching from PBCs to SBCs lead to density deviations in the boundary region. Thus, the staggered coupling algorithm as introduced in Section 3 is employed, using a very soft FE part, i.e. the Young's modulus chosen is very low $(E=0.1 \mathrm{MPa})$ and Poisson's ratio is set to zero. Furthermore, no Dirichlet boundary conditions are applied to the FE domain, except those required to avoid rigid body motion. Moreover, a simulation time of $0.5 \mathrm{~ns}$ in each MD-FE iteration step is chosen for the MD system.

The diagonal elements of the stress tensor in the MD domain were calculated with the help of the anchor point forces. They were obtained by collecting the components of the anchor point forces that are perpendicular to the surface of the box divided by the area of the surface, except for a region of $1.5 \mathrm{~nm}$ width at the margin of the simulation box which is equal to the thickness of the boundary region. Figure [1] shows the three diagonal elements of the stress tensor as a function of the number of MD-FE iterations. All three components have the same behaviour. They start at $6000 \mathrm{kPa}$ and finally converge to zero. The stress fluctuates around zero after 125 iterations which means that the anchor points are in the equilibrium positions. The final configuration of this step was used for the next step to apply a deformation to the box. During the initial equilibration the size of the box did not change too much and the density in the MD region has been reduced by only $0.1 \%$.

\subsection{Convergence criteria}

In order to investigate the performance of the coupling procedure, a pure FE cube is used as a reference system. The overall edge length and the discretization is chosen identical to the FE part of the coupled 


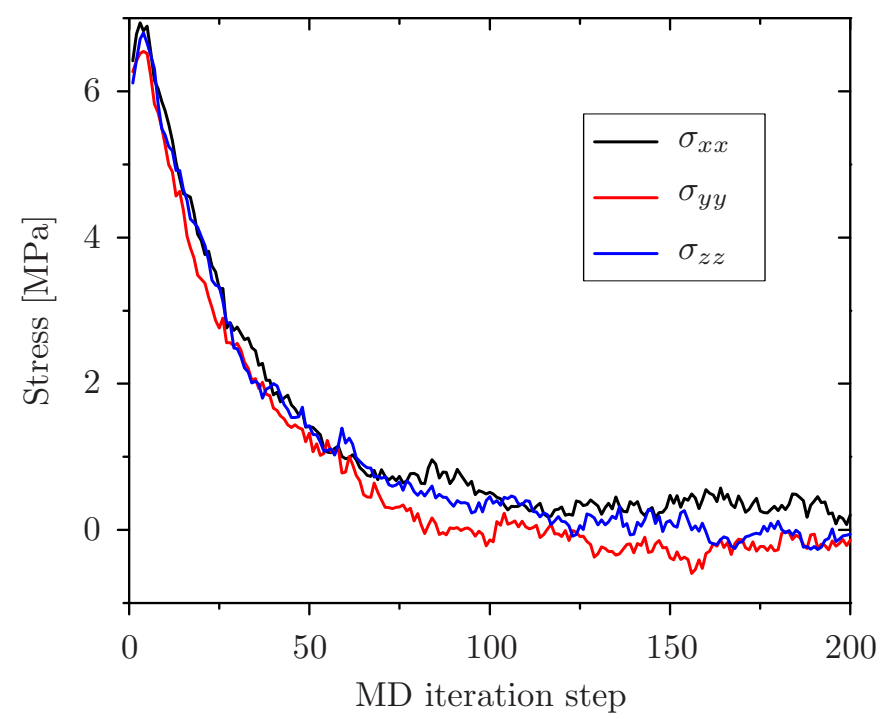

Figure 11: Three diagonal elements of stress tensor which were obtained from the anchor point forces in the MD-FE equilibration procedure.

system. Both the coupled and the pure FE cube are subjected to the same boundary conditions and material parameters. Thus, the reaction forces due to Dirichlet boundary conditions and the Cauchy stresses at the surfaces can be compared to each other. The reaction forces at the surface of the coupled system are defined as

$$
\mathbf{f}_{c p l}^{e x t}=\left\{\mathbf{f}_{c I}^{e x t}\right\} \quad I \text { : node index at the surfaces, }
$$

while the reaction forces at the surfaces of the pure FE system are introduced as $\mathbf{f}_{F E}^{\text {ext }}$. The relative deviation between reaction forces of the coupled and the pure FE system is defined as:

$$
e_{j}^{f}=\frac{\sum_{I}\left|f_{c p l I j}^{e x t}-f_{F E I j}^{e x t}\right|}{\sum_{I}\left|f_{F E I j}^{e x t}\right|}, \quad j=x, y, z, \quad I \text { : node index at the surfaces }
$$

In (56), the sum of nodal deviations in reaction forces is divided by the sum of reaction forces computed in the pure FE simulation. In order to avoid neutralization of positive and negative contributions, the absolute values are employed to define $e_{j}^{f}$. Similarly, the deviation between Cauchy stresses is introduced as

$$
e_{j k}^{\sigma}=\frac{\sum_{I}\left|\sigma_{c p l j k}\left(\mathbf{X}_{I}\right)-\sigma_{F E j k}\left(\mathbf{X}_{I}\right)\right|}{\sum_{I}\left|\sigma_{F E j k}\left(\mathbf{X}_{I}\right)\right|}, \quad j, k=x, y, z, \quad I: \text { node index at the surfaces }
$$

which considers each node at the surfaces. Again, the nodal contributions of stress deviations are evaluated with respect to the stresses of the pure FE cube. Nonzero values $e_{j}^{f}$ and $e_{j k}^{\sigma}$ indicate differences in the description accessible by a bare continuum model and the coupled system under consideration of particle effects. In the following we will concentrate on deviations in the $y$-direction in which the system is stretched: thus we will focus on $e_{y}^{f}$ and on $e_{y y}^{\sigma}$. With these measures at hand, convergence criteria can be formulated which are based on the comparison to a pure FE system:

a) Convergence in the deviation of the reaction forces in the $y$-direction at iteration step $i$ :

$$
\Delta e_{y(i)}^{f}=e_{y(i)}^{f}-e_{y(i-1)}^{f} ; \quad\left|\Delta e_{y(i)}^{f}\right|<t o l_{a}
$$

b) Convergence in the deviation of the Cauchy stress $\sigma_{y y}$ at iteration step $i$ :

$$
\Delta e_{y y(i)}^{\sigma}=e_{y y(i)}^{\sigma}-e_{y y(i-1)}^{\sigma} ; \quad\left|\Delta e_{y y(i)}^{\sigma}\right|<t o l_{b}
$$


Convergence of a coupled simulation is reached if one (or both) of these conditions is met, i.e. if $\Delta e_{y(i)}^{f}$ or $\Delta e_{y y(i)}^{\sigma}$ are lower than a predefined threshold, see (58) and (58).

In addition to these criteria, which require comparison to a reference system, break conditions can be introduced that use data inherent to the system under consideration. For example, the mean value of the Cauchy stress $\sigma_{j k}$ at surface nodes (number: $n_{I}$ ) of the FE cube can be computed:

$$
\bar{\sigma}_{j k}=\frac{1}{n_{I}} \sum_{I} \sigma_{c p l ~ j k}\left(\mathbf{X}_{I}\right), \quad j, k=x, y, z, \quad I: \text { node index at the surfaces }
$$

Consequently, a third convergence criterion for the investigations in this contribution is the relative difference of $\bar{\sigma}_{y y}$ in two subsequent iteration steps:

c) Convergence in $\bar{\sigma}_{y y}$ at iteration step $i$ :

$$
\Delta \bar{\sigma}_{y y(i)}=\frac{\bar{\sigma}_{y y(i)}-\bar{\sigma}_{y y(i-1)}}{\bar{\sigma}_{y y(i-1)}} ; \quad\left|\Delta \bar{\sigma}_{y y(i)}\right|<t_{c} l_{c}
$$

In analogy to the cases discussed above, convergence is reached if $\left|\Delta \bar{\sigma}_{y y(i)}\right|$ is smaller than $t_{c} l_{c}$.

The suitability of these criteria will be discussed subsequently at the example of a polystyrene system coupled to FE. The parameters are:

$\begin{array}{ll}\text { Young's modulus (FE) } & E=800 \mathrm{MPa} \\ \text { Poisson's ratio (FE) } & \nu=0.3 \\ \begin{array}{l}\text { spring constant } \\ \text { (between anchor points and MD beads) }\end{array} & k_{I}=2.32 \frac{\mathrm{N}}{\mathrm{m}} \\ \text { weighting factor } & \alpha=0.5 \\ \text { strain } & \varepsilon_{y y}=1 \% \\ \text { number of anchor points } & n^{M S}=9004 \\ \text { number of FE nodes } & 936 \\ \text { number of nodes in } & \\ \begin{array}{l}\text { Lagrangian multiplier space } \\ \text { total number of DOFs }\end{array} & 448 \\ L_{2} \text {-norm } & 31164\end{array}$

The discretization of the Lagrangian multipliers is the same as for the geometry of the continuum and for the displacements. In the system under consideration, the boundary region contains $448 \mathrm{FE}$ nodes as well as the same number of nodes in the Lagrangian multiplier space. Furthermore, the pure FE system is computed with the same Young's modulus and Poisson's ratio, but consists of 1000 FE nodes.

In Figure 12, the normal stresses in $y$-direction are plotted for a pure FE simulation, cf. b) and c), as well as for a coupled simulation, cf. d) and e). In order to demonstrate the deformation behaviour, displacements are scaled by 100 in c) and e). As expected, the pure FE cube deforms to a cuboid and shows a uniform normal stress distribution $\sigma_{F E} y y=E \varepsilon_{y y}=8.0 \mathrm{MPa}$. In case of the coupled simulation, the deformed configuration is very similar to a cuboid, while the stress distribution is not longer uniform. In the vicinity of the boundary region, the stress of the coupled system is lower (lowest value approximately 3.60 MPa). This observation is discussed and explained in more detail in Section 4.3. In contrast, the stress is very close to that of the pure FE domain in the centre of the $x z$-surface (approximately $7.98 \mathrm{MPa}$ ). Furthermore, the stress at the $y z$ - and $x y$-surfaces is distributed quite uniformly and does not deviate much from that of the pure FE system. In view of a quantitative investigation, the relative deviation between the stress distributions of pure and coupled simulations is introduced:

$$
\delta \sigma_{j k}\left(\mathbf{X}_{I}\right)=\frac{\sigma_{c p l j k}\left(\mathbf{X}_{I}\right)-\sigma_{F E j k}\left(\mathbf{X}_{I}\right)}{\sigma_{F E j k}\left(\mathbf{X}_{I}\right)}, \quad j, k=x, y, z, \quad I: \text { node index at the surfaces }
$$



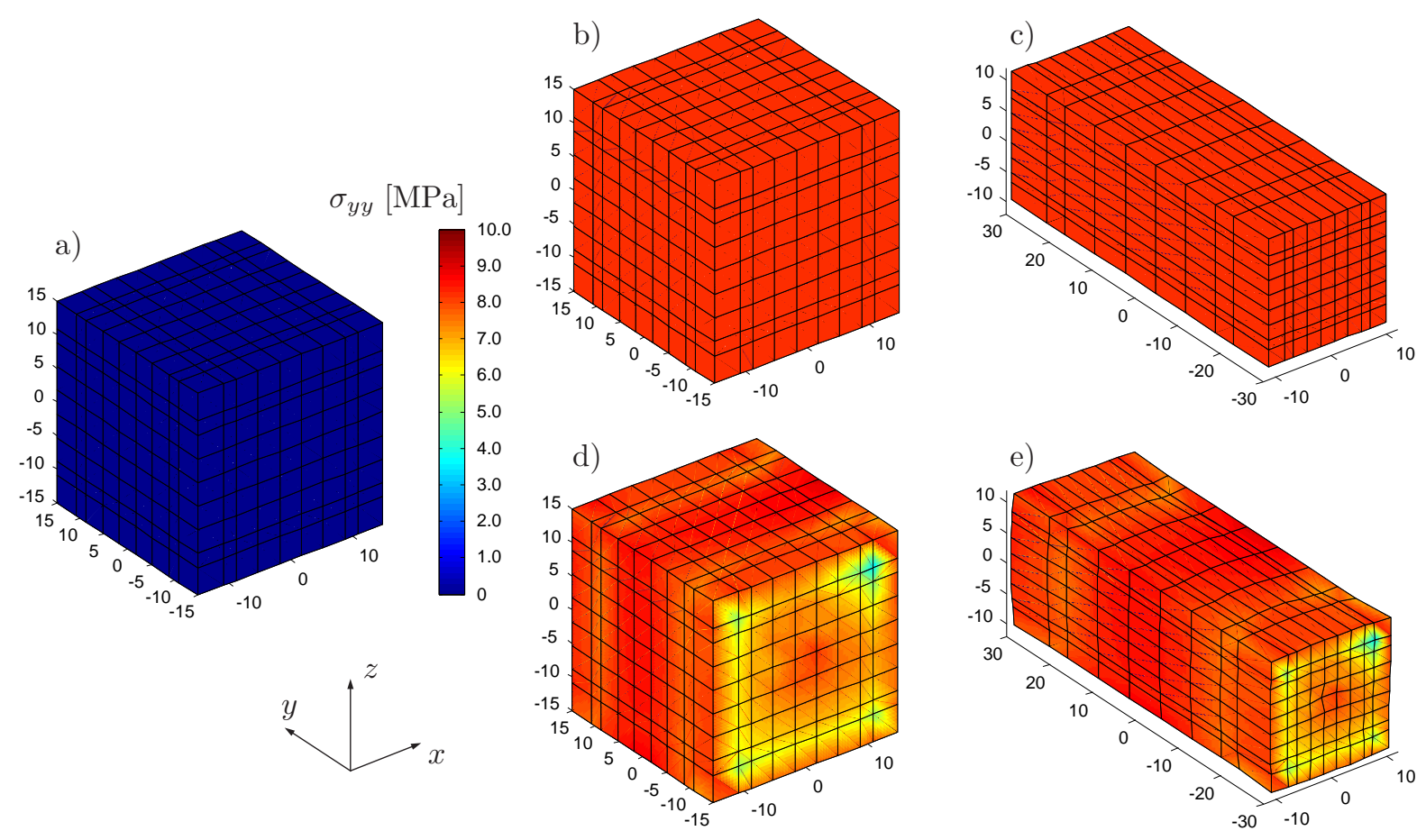

Figure 12: Uniaxial tension test, comparison of pure FE (b, c) and coupled MD-FE (d, e) simulation: $E=800 \mathrm{MPa}, \varepsilon_{y y}=1 \%$, after 100 iteration steps between $\mathrm{MD}$ and $\mathrm{FE}$, the unit of stress is [MPa], the unit of length is [nm]: a) undeformed system, b) and c) normal stress $\sigma_{F E} y y$ at the external surface of the deformed FE system, d) and e) normal stress $\sigma_{c p l ~ y y}$ at the external surface of the deformed $\mathrm{FE}$ system; in c) and e) the displacements are scaled by 100 .
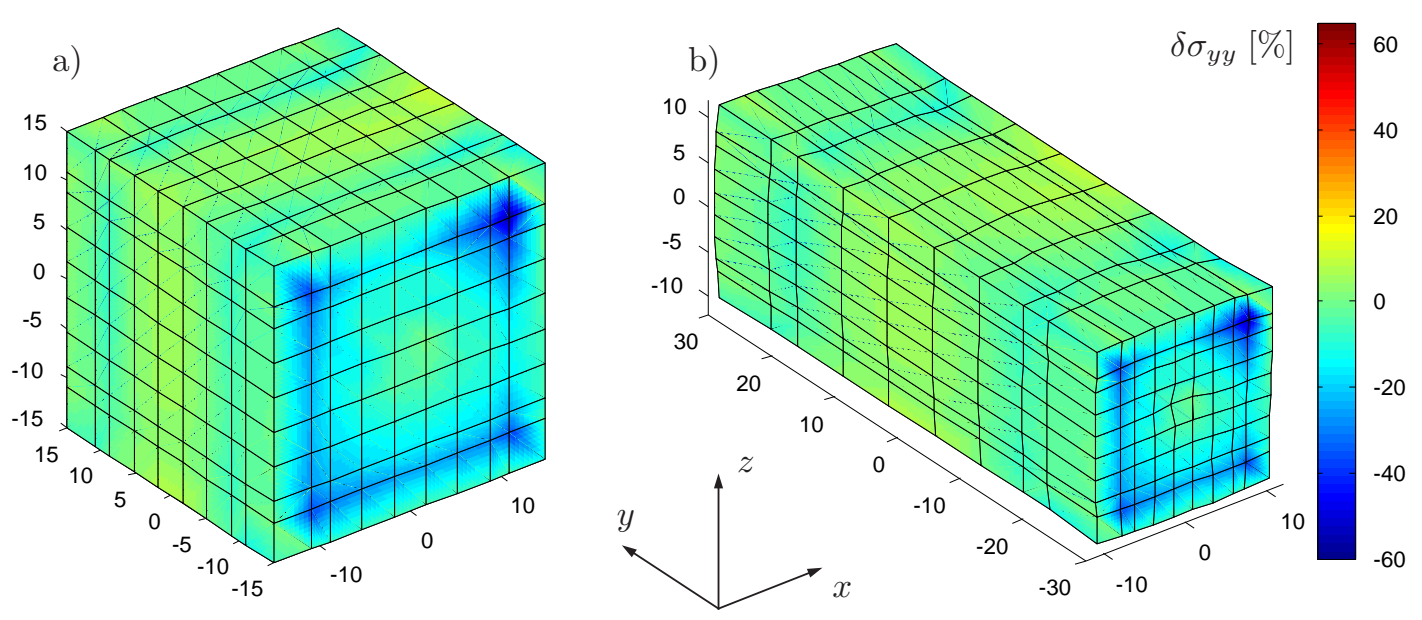

Figure 13: Uniaxial tension test, $E=800 \mathrm{MPa}, \varepsilon_{y y}=1 \%$, after 100 iteration steps between MD and FE: deviation of normal stress $\delta \sigma_{y y}[\%]$ at the external surface of the FE domain, a) actual deformation, b) displacements scaled by 100

Deviation $\delta \sigma_{y y}$ is plotted in Figure 13. In accordance with Figure 12 a non-negligible deviation is observed exclusively near the bridging domain, especially at the corners, whereas much lower values are obtained for rest of the system.

Furthermore, convergence criterion a), cf. (58), is given in Figure 14, the initial deviation of 227.6\% decreases to $12.8 \%$, while $\Delta e_{y}^{f}$ amounts to only $0.058 \%$ after $100 \mathrm{MD}-\mathrm{FE}$ iteration steps. Thus, the system does not change significantly any more and convergence can be assumed.

Roughly the same behaviour is obtained in case of convergence criterion b), cf. (59) and Figure 15. Here, the initial deviation is $123.0 \%$ and decreases to $8.0 \%$. After $100 \mathrm{MD}-\mathrm{FE}$ iteration steps, $\Delta e_{y y}^{\sigma}$ is 

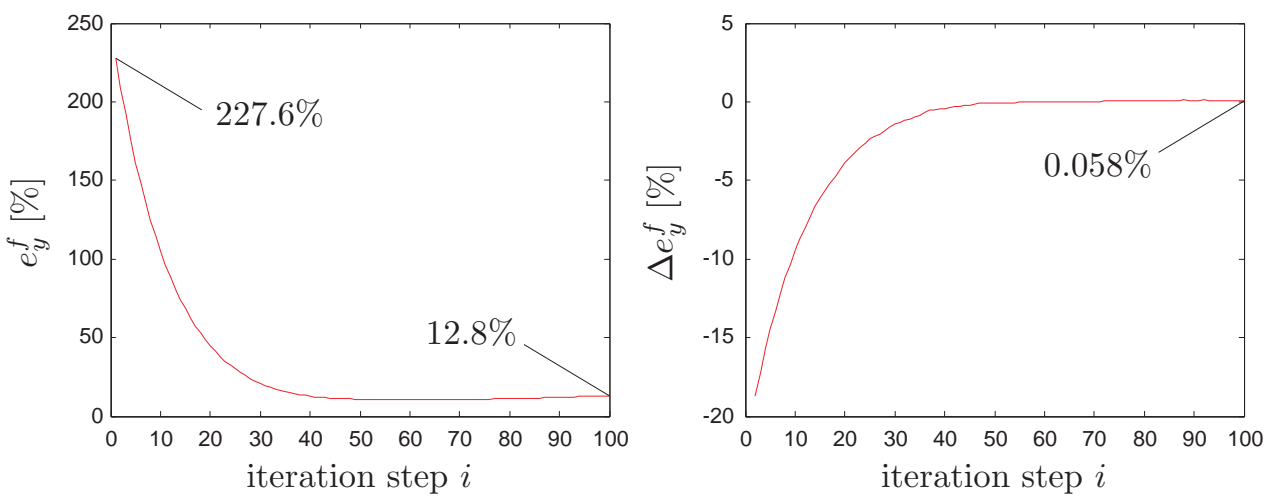

Figure 14: Coupled simulation, uniaxial tension test, $E=800 \mathrm{MPa}, \varepsilon_{y y}=1 \%$ : relative deviation of reaction forces $e_{y}^{f}$ and convergence behaviour of $\Delta e_{y}^{f}$ versus MD-FE iteration step $i$
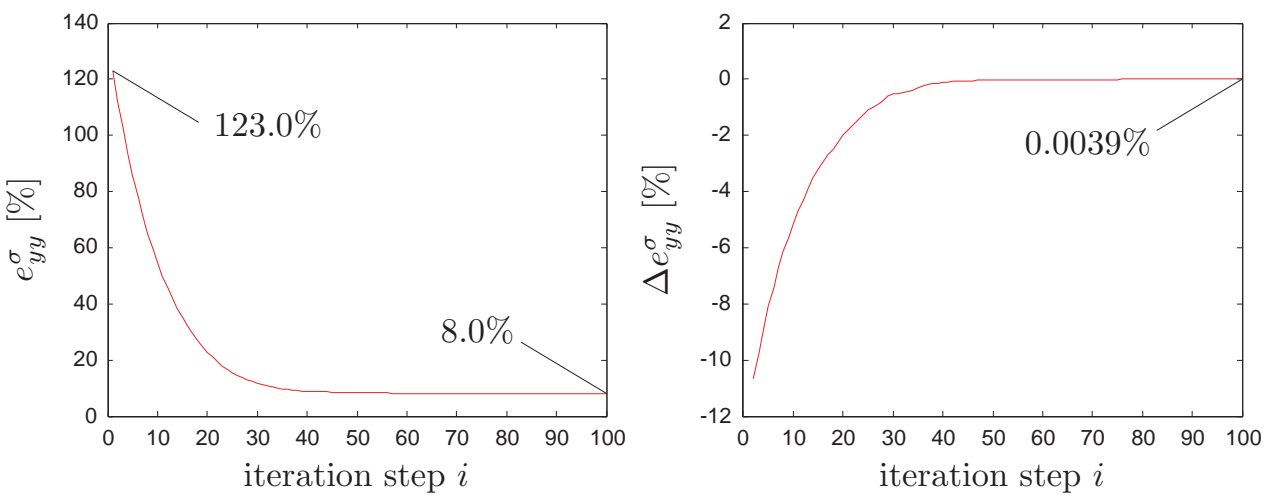

Figure 15: Coupled simulation, uniaxial tension test, $E=800 \mathrm{MPa}, \varepsilon_{y y}=1 \%$ : deviation of normal stress $e_{y y}^{\sigma}$ and convergence behaviour of $\Delta e_{y y}^{\sigma}$ versus MD-FE iteration step $i$

$0.0039 \%$.

In contrast to the criteria discussed above, convergence of the system can be obtained independently of a pure FE simulation by evaluating $\bar{\sigma}_{y y}$ as well as $\Delta \bar{\sigma}_{y y}$, cf. (60) and (61). The results are plotted in Figure 16 starting at $15.81 \mathrm{MPa}, \bar{\sigma}_{y y}$ decreases to $7.56 \mathrm{MPa}$ after 100 iteration steps. Compared to a pure $\mathrm{FE}$ simulation with a constant value of $8.00 \mathrm{MPa}$, which is equal to the analytic result, there is a relative deviation of approximately $5.5 \%$. After 100 iteration steps $\bar{\sigma}_{y y}$ amounts to only $-0.024 \%$ which makes it a suitable convergence criterion since it does not require comparison to a pure FE system.
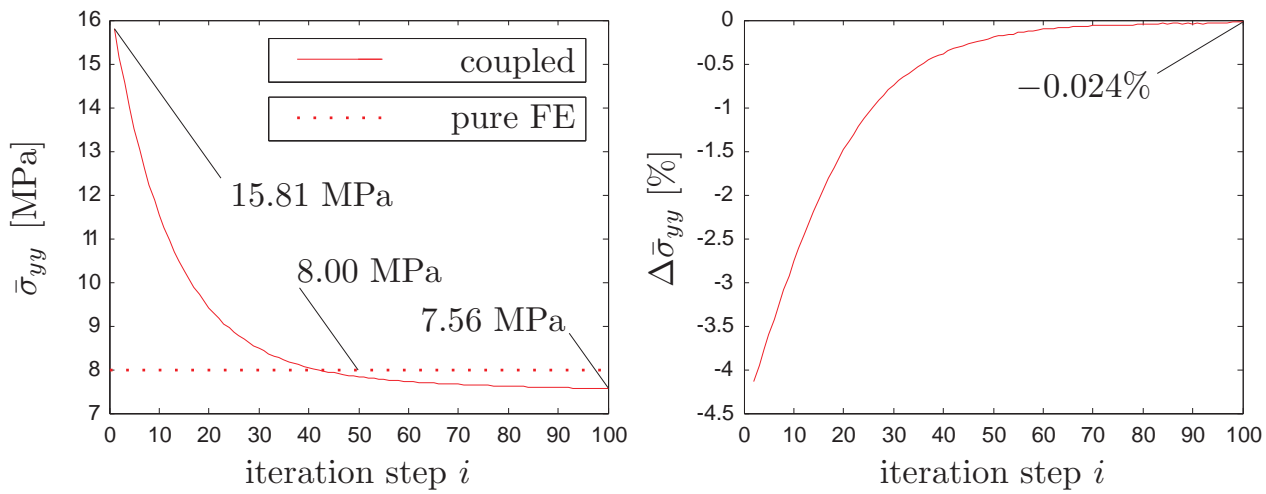

Figure 16: Uniaxial tension test, $E=800 \mathrm{MPa}, \varepsilon_{y y}=1 \%$ : mean value of normal stress $\bar{\sigma}_{y y}$ (coupled and pure FE simulation) and convergence behaviour of $\Delta \bar{\sigma}_{y y}$ versus MD-FE iteration step $i$ 
After 100 iteration steps, $\left|\Delta \bar{\sigma}_{y y}\right|$ is significantly lower than $0.1 \%$, thus, the number of iteration steps chosen for this investigation seems to be reasonable. Consequently, at least 100 iteration steps are computed for each system and the convergence is checked based on a comparison to a reference system by using criteria a) and b) as well as c). All three criteria are used in consideration of the following reasons:

- Criterion a) is based on reaction forces as a direct output of the simulation; due to the comparison to a reference system, performance and results of the coupled computation can be investigated and evaluated;

- Criterion b) allows for a better understanding and visualization of the deviations between the coupled and the pure FE system, especially in case of uniaxial tension tests;

- Criterion c) does not depend on the comparison to a reference system. Thus, it is a well suited criterion for systems that cannot be computed using a pure FE approach, which is exactly the intention of the coupling method;

Note that the focus of this contribution lies on the methodology and performance of the coupling method proposed. This requires a quantification of convergence properties which can be evaluated using the criteria given above. Nevertheless, this work does not yet aim at an optimized specification of the threshold parameters $t o l_{a}, t_{b} l_{b}$, and $t o l_{c}$, which would necessitate much more experience in the application of the method. For example, a number of systems at different load cases would have to be investigated in order to define reliable thresholds. Based on the findings presented in this section, we decided instead, that a number of $100 \mathrm{MD}-\mathrm{FE}$ iteration steps is a reasonable choice for the first investigations. This ensures an acceptable compromise between convergence and computation times.

\subsection{Example 1: coupled simulation of pure polystyrene}

In this section, we systematically investigate a system of pure polystyrene coupled to a finite element domain. The main focus is on the influence of Young's modulus on the coupled simulation and on the observed differences to the pure FE system. The parameters are the same as introduced in Section 4.1. except Young's modulus which is varied from $400 \mathrm{MPa}$ to $1500 \mathrm{MPa}$ on the FE side. The Young's modulus on the MD side is constant $827 \mathrm{MPa}$.

Firstly, the deformation behaviour of the coupled system is investigated qualitatively. To this end, the actual displacements of the FE nodes are scaled by factor of 100. In Figure 17 the deformations of the pure polystyrene system are plotted together with deviation $\delta \sigma_{y y}$ of the normal stresses in $y$ direction. In case of $E=400 \mathrm{MPa}$, the particle domain is stiffer than the finite element part which, on the one hand, causes higher normal stresses in the centre of the $x z$-surfaces and, on the other hand, less contraction in the centre of the system. Both effects are clearly visible in Figure 17, whereby positive values of $\delta \sigma_{y y}$ express higher stresses in the coupled simulation due to definition (62). For $E=1500 \mathrm{MPa}$, the particle domain is significantly softer than the continuum, which leads to more contraction in the centre and lower stresses in the central $x z$-surfaces. The smallest deviation $\delta \sigma_{y y}$ as well as the most uniform contraction perpendicular to the load direction are obtained for Young's moduli between $E=600 \mathrm{MPa}$ and $E=900 \mathrm{MPa}$.

In order to quantify these observations, the deviations of reaction forces $e_{y}^{f}$ and of normal stress $e_{y y}^{\sigma}$ are investigated. In Figure 18, $e_{y}^{f}$ and $e_{y y}^{\sigma}$ are plotted as a function of $E$ : qualitatively, both curves show the same behaviour. A minimum for $E=600 \mathrm{MPa}$ is observed in both cases. At $E=400 \mathrm{MPa}$, $e_{y}^{f}$ is $9.40 \%$ and decreases with larger Young's moduli to a minimum of $6.80 \%$ at $E=600 \mathrm{MPa}$. After that, $e_{y}^{f}$ increases and becomes $11.80 \%$ at $E=1500 \mathrm{MPa}$. It is expected that a further increase of $e_{y}^{f}$ would be observed for Young's moduli smaller than $E=400 \mathrm{MPa}$ or larger than $E=1500 \mathrm{MPa}$. Very similar, although at a higher level, $e_{y y}^{\sigma}$ is $14.0 \%$ at $E=400 \mathrm{MPa}$, decreases to its minimum of $8.90 \%$ at $E=600 \mathrm{MPa}$, and increases to $22.80 \%$ at $E=1500 \mathrm{MPa}$.

These results support the qualitative findings discussed above, where the best fit between coupled and FE reference simulation has been observed for $E$ between $600 \mathrm{MPa}$ and $900 \mathrm{MPa}$. Furthermore, this is in line and of the same order of magnitude obtained from the parameter identification of a pure MD simulation, see Section 4.1. There, a Young's modulus of $E=827 \mathrm{MPa}$ has been determined, which, however, is not exactly the same.

In order to understand this stiffness deviation, the particle system as well as the coupling parameters used here should be considered carefully. For sure, they have a strong influence on the performance and the results of the coupled simulation. First qualitative hints can be obtained from Figure 17 in case of 

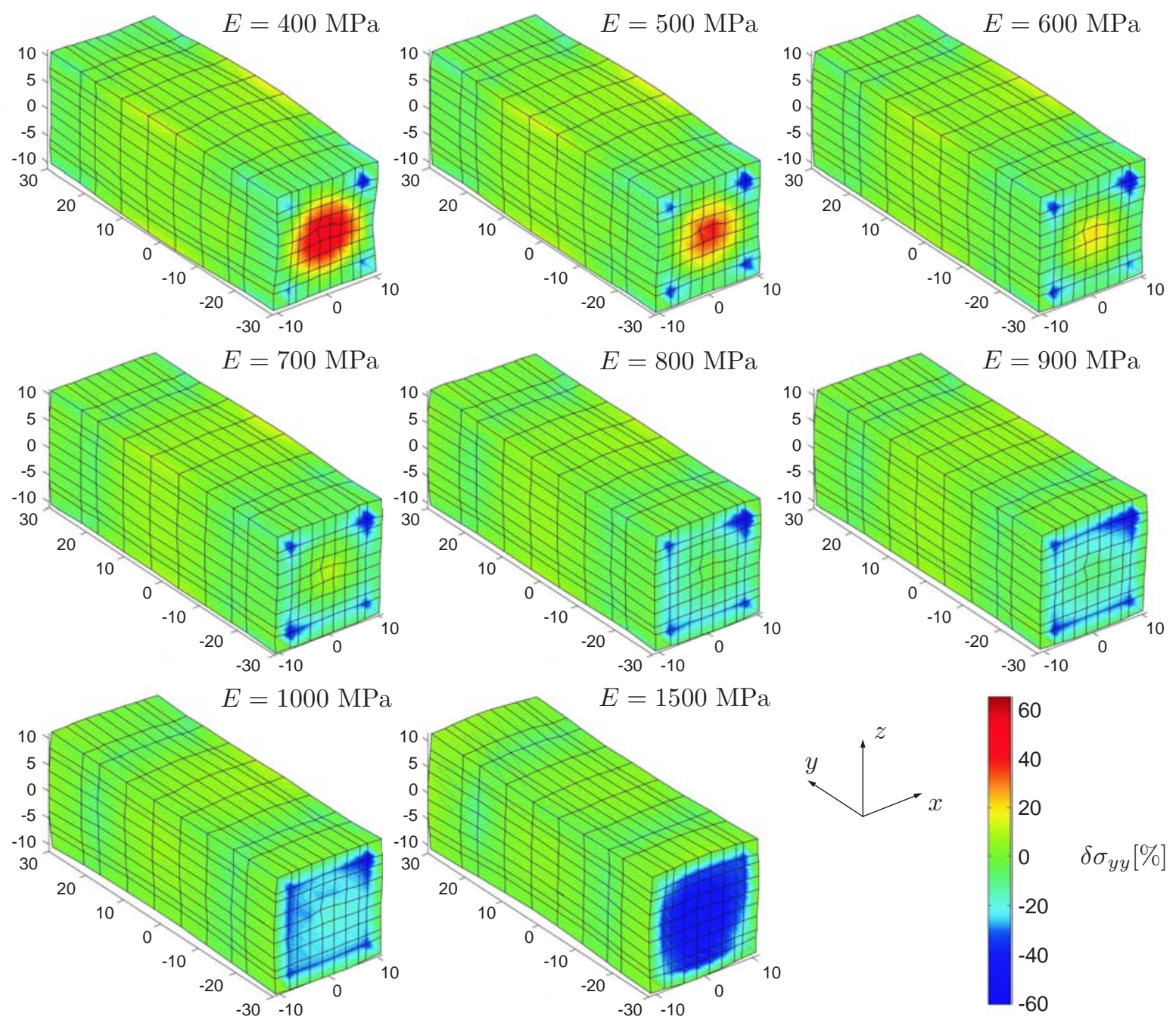

Figure 17: Deviation $\delta \sigma_{y y}$ of the normal stress $\sigma_{y y}$ of polystyrene, coupled to an FE domain, $\delta \sigma_{y y}$ is shown at the external surface of the FE domain; the Young's modulus of the FE part varies from $400 \mathrm{MPa}$ (top left) to $1500 \mathrm{MPa}$ (bottom centre), the displacements are scaled by 100

a Young's modulus between $E=600 \mathrm{MPa}$ and $E=900 \mathrm{MPa}$, quite a uniform distribution of deviations in the normal stress is obvious from the plots, even at the $x z$-surfaces. Exceptions are the corners near the bridging domain. Here, the stress deviation differs significantly which could be caused by the choice of coupling parameters, e.g. the weighting function $\alpha$, but, most likely, also by the geometry of the system. Due to the cubic shape of the particle domain, sharp edges and corners occur, which, most likely, exert considerable influences on the local behaviour of the particle system in these regions. The systematic investigation of these factors and a discussion of possible solution strategies are postponed to later contributions since this would exceed the scope of this work. Nevertheless, from the molecular dynamics point of view, certain reasons for the stress deviations in the bridging domain, and especially nearby the corners, can be identified already here. The polymer chains in the effective boundary region supposedly have a different effective elastic characteristic than those in the pure MD domain. Close to the corners, the polymer is in contact with three adjacent artificial interfaces, and the stochastic boundary conditions as well as the anchor points are surely confining the dynamics there. Furthermore, also the DPD region is probably influencing the elastic properties. It thus cannot be expected that the polymer exhibits the same behaviour in this region as in the pure MD part. Most likely, a possibly more relevant contribution to this observation may be the polymer chain length in the effective boundary region. By construction of the MD box, the average chain length in the boundary region is shortened to 8 monomers per chain, which is rather small compared to the pure MD region where it amounts to 200 monomers per chain. At the interface to the FE domain and, in particular, in the vicinity of 


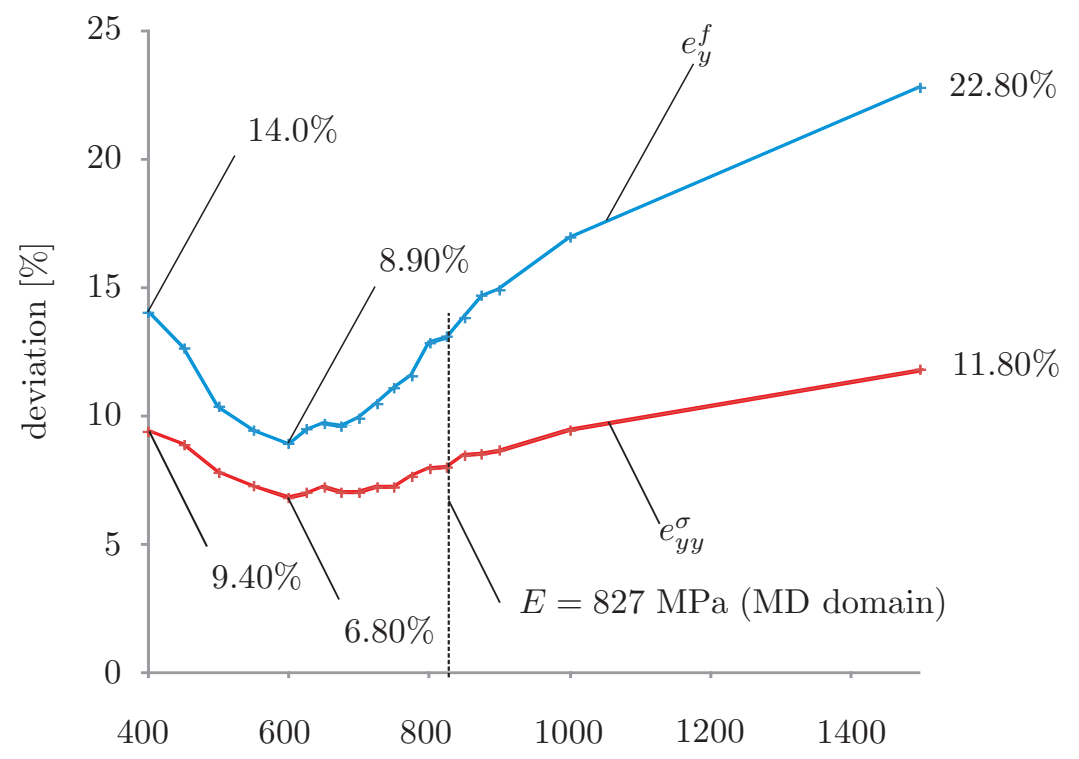

Young's modulus $E[\mathrm{MPa}]$ in $\mathrm{FE}$ domain

Figure 18: Coupled simulation of polystyrene: deviation of normal stress $e_{y y}^{\sigma}$ and reaction forces $e_{y}^{f}$ as a function of the Young's modulus of the FE domain, evaluated after $100 \mathrm{MD}-\mathrm{FE}$ iteration steps

the sharp edges of the boundary domain the chain length is reduced further. Some chains are even shorter than the entanglement length, which significantly influences the elastic properties of polymers 33, 34: Young's modulus usually increases with the chain length. This is in line with the qualitative findings in Figure 17, near the corners of the bridging domain, $\delta \sigma_{y y}$ is negative for all Young's moduli considered. This implies the particle domain being softer than the FE domain, which fits well to the reduced chain length in this region. Consequently, the MD system as a whole behaves softer than its pure FE counterpart. This leads to a reduced stiffness for the entire coupled system. Hence, comparing the coupled system's response to that of a pure FE system, minimum deviations of normal stress $e_{y y}^{\sigma}$ and reaction forces $e_{y}^{f}$ are obtained at a lower Young's modulus. This is evident from Figure 18, where the minima of $e_{y y}^{\sigma}$ and $e_{y}^{f}$ occur at $E=600 \mathrm{MPa}$, although $E=827 \mathrm{MPa}$ has been identified from the pure MD system under PBC which avoid the aforementioned artefacts. Future activities to resolve the discrepancies will be discussed briefly in Section 5 .

Finally, the convergence behaviour is investigated by comparing the criteria introduced above. All systems have been computed for $100 \mathrm{MD}-\mathrm{FE}$ iteration steps, not yet employing any break condition based on certain thresholds. Instead, the convergence criteria are evaluated at iteration step 100 and presented as a function of the Young's modulus of the FE domain, cf. Table 1. It is observed that all values of $\left|\Delta e_{y(100)}^{f}\right|,\left|\Delta e_{y y(100)}^{\sigma}\right|$, and $\left|\Delta \bar{\sigma}_{y y(100)}\right|$ are significantly lower than $1 \%$, which is very promising. Thus, all systems have reached an equilibrium configuration, i.e. convergence can be stated. Furthermore, $\Delta e_{y(100)}^{f}$ and $\Delta e_{y y(100)}^{\sigma}$ show the lowest absolute values for Young's moduli between $E=600 \mathrm{MPa}$ and $E=900 \mathrm{MPa}$, which is the same range as for the stress deviations in Figure 17 . Additionally, the lowest values of $e_{y}^{f}$ and $e_{y y}^{\sigma}$ lie in the same interval, cf. Figure 18. Pending further investigations, it can be assumed that there is a certain relation between the convergence behaviour and the lowest deviation with respect to a pure FE simulation. However, further decrease of $\left|\Delta e_{y(100)}^{f}\right|$ and $\left|\Delta e_{y y(100)}^{\sigma}\right|$ values for higher Young's moduli have to be noted as well. This is caused probably by the fact that the stiffness of the system is increasingly dominated by the FE region when the Young's modulus is chosen larger while the influence of the particle region is reduced. Thus, we assume that the system's response becomes more similar to the pure, static FE system which requires less MD-FE iteration steps to reach any convergence criterion. From our point of view, the same reason can be assumed for the decrease of $\left|\Delta \bar{\sigma}_{y y}(100)\right|$ with increasing Young's moduli.

With these results at hand, the particle system is now replaced by polystyrene containing a single silica nanoparticle. 
Table 1: Coupled simulation of pure polystyrene: convergence criteria evaluated after 100 MD-FE iteration steps for different FE-Young's moduli $E$

\begin{tabular}{|r||r|r|r|}
\hline$E[\mathrm{MPa}]$ & $\Delta e_{y(100)}^{f}[\%]$ & $\Delta e_{y y(100)}^{\sigma}[\%]$ & $\Delta \bar{\sigma}_{y y(100)}[\%]$ \\
\hline \hline 400 & -0.1687 & -0.0765 & -0.0909 \\
\hline 500 & -0.0694 & -0.0386 & -0.0581 \\
\hline 600 & -0.0140 & -0.0288 & -0.0422 \\
\hline 700 & 0.0356 & -0.0038 & -0.0338 \\
\hline 800 & 0.0578 & 0.0039 & -0.0236 \\
\hline 900 & 0.0547 & 0.0058 & -0.0233 \\
\hline 1000 & 0.0528 & 0.0167 & -0.0234 \\
\hline 1500 & 0.0259 & 0.0118 & -0.0076 \\
\hline
\end{tabular}

\subsection{Example 2: coupled simulation of a nanocomposite}

In this section, the system under consideration is polystyrene enclosing a single silica nano-filler particle. The interaction parameters used for the polystyrene part are the same as described in Section 4.3 For silica we have used a setup that had been adopted already in a CG MD study of PS-silica nanocomposites 35 . In the mapping scheme of silica a $\mathrm{SiO}_{2}$ unit is represented by a CG bead, the centre of which is located at the Si site. The nanoparticle of a $4 \mathrm{~nm}$ diameter is defined by 873 beads. The interaction potentials between polystyrene and the silica nanoparticle were again obtained by Iterative Boltzmann Inversion 21. The surface beads of the nanoparticle have a dominant contribution to the interaction with the polymer matrix. The interaction of the polymer with the inner core beads is weaker. This behaviour is considered by different interaction potentials between PS and surface beads as well as between PS and core beads. The mapping scheme, the method to develop the CG potential and also the validation of the CG potential were explained with more detail in [35. A qualitative representation of the nanocomposite is shown in Figure 19. The filler particle is located in the centre of the particle region; it is surrounded by the PS matrix. The volume fraction of the nanoparticle is approximately $0.6 \%$, which is rather small. Nevertheless, this seems to be sufficient to estimate the capability of the new MD-FE coupling technique in such inhomogeneous systems which cannot be treated by pure FE methods with an accuracy as accessible by particle-based computations. Coupled simulations with an enlarged volume fraction of the nanoparticle should, in principle, cause no computational problems. The model parameters of the nanocomposite such as temperature, pressure, length and number of the polymer chains, etc. are the same as adopted in the pure PS example.

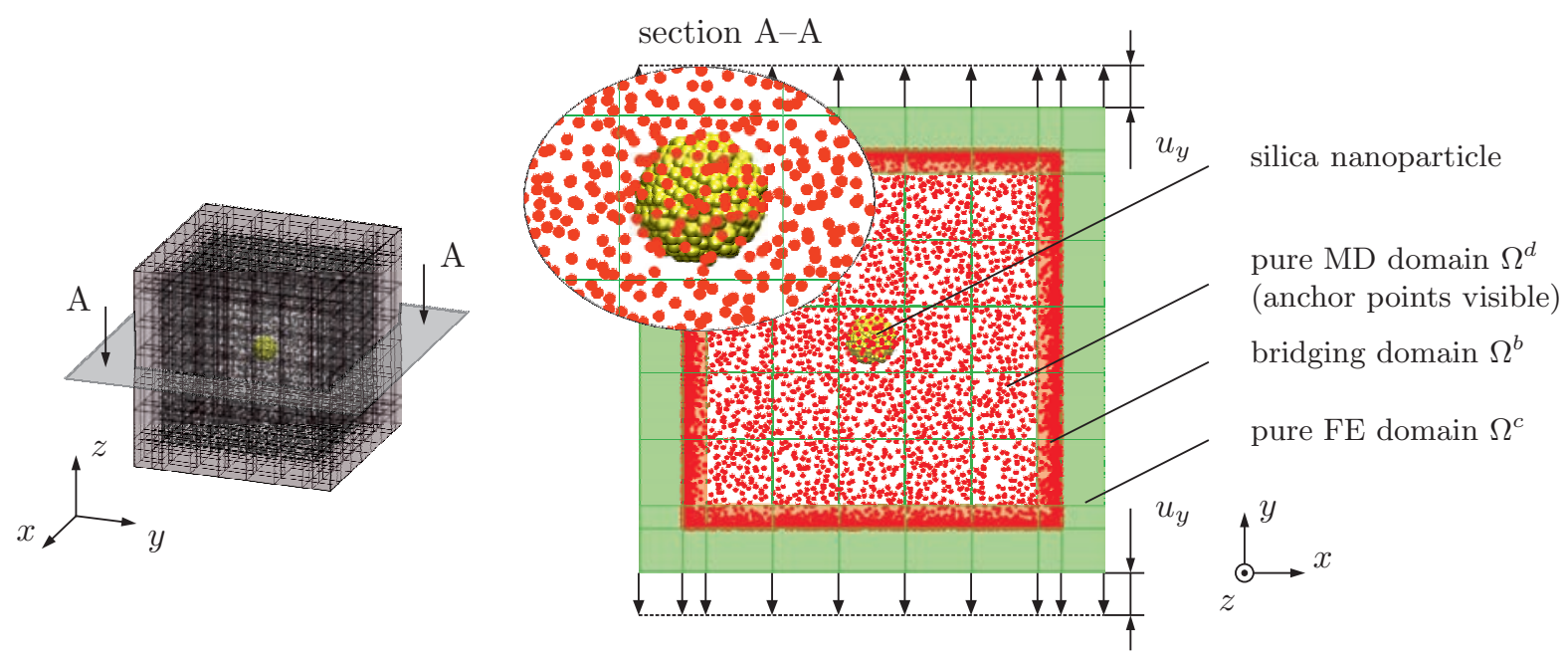

Figure 19: Coupled system with silica nanoparticle subjected to prescribed displacements $u_{y}$ at the upper and lower $x z$-surfaces; for proper visualization, only the anchor points and the silica particle are plotted schematically, $3 \mathrm{~d}$ view (left) and sectional view $\mathrm{A}-\mathrm{A}$ (right) 
For the nanocomposite the same simulations have been carried out as for pure polystyrene. Due to the small volume fraction, no qualitative differences have been obtained, only small quantitative deviations occurred. Thus, we do not discuss these results in detail. In extension to the previous simulations, the system is now subjected to several load steps, each of $1 \%$ in order to investigate the performance beyond the approximately linear elastic region. Due to the small volume fraction of the filler particle, the Young's modulus of the MD domain remains at $E=827 \mathrm{MPa}$ which is the same value as obtained for the pure PS system.

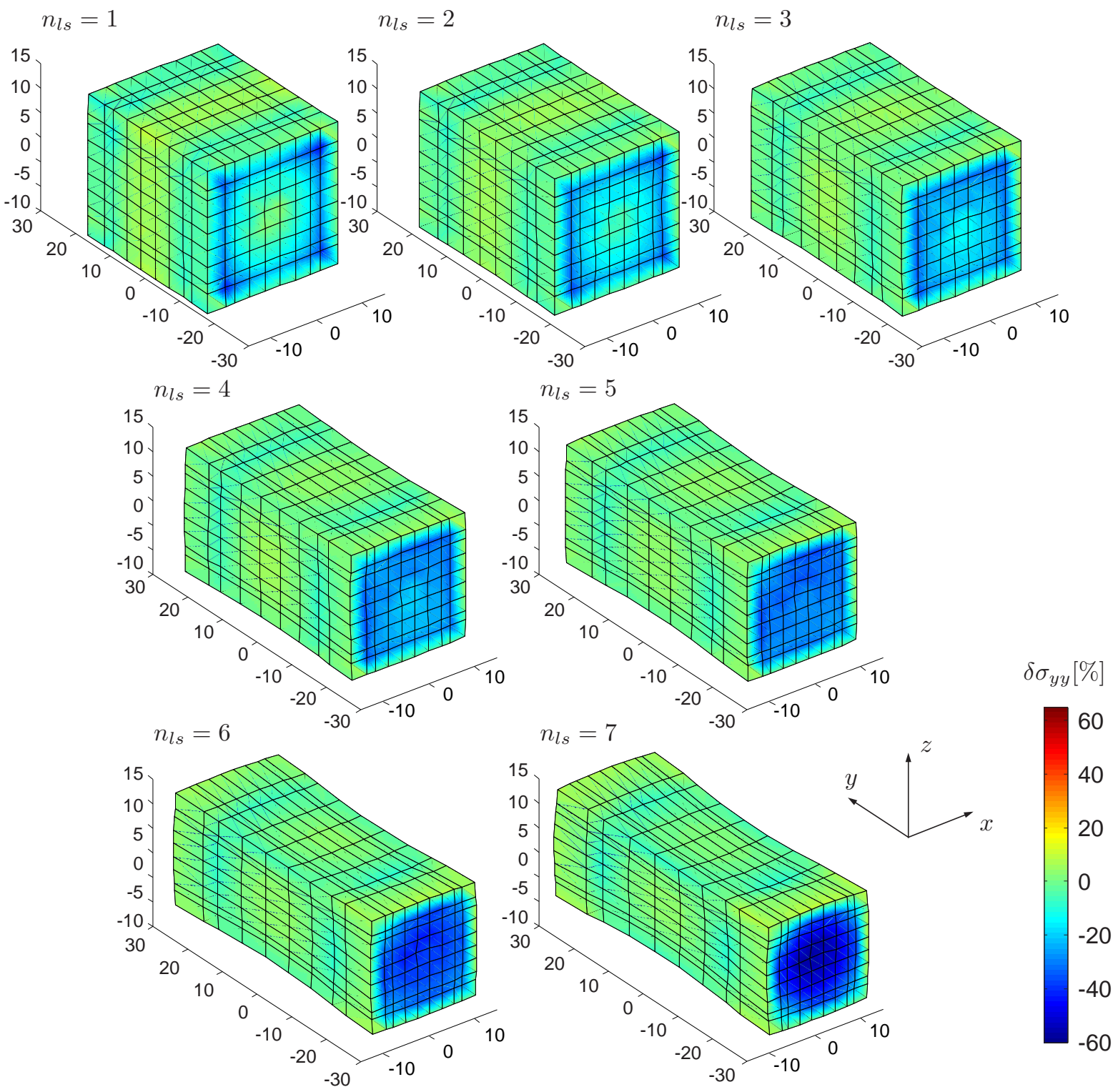

Figure 20: Coupled simulation of polystyrene with a silica nanoparticle; Young's modulus of the FE system: $E=800 \mathrm{MPa}$, number of load steps: $n_{l s}=7$, number of MD-FE iteration steps per load step: $n_{M D}=100$; deviation $\delta \sigma_{y y}$ of normal stress $\sigma_{y y}$ at the external surface of the FE domain plotted after each load step, displacements are scaled by factor of 15

In Figure 20 the normal stress deviations $\delta \sigma_{y y}$ are plotted at the surfaces of the FE domain in case of $E=800 \mathrm{MPa}$. Due to the findings of the pure PS system it can be expected that the minimum deviations $e_{y}^{f}$ and $e_{y y}^{\sigma}$ would be obtained for Young's moduli of the FE system significantly lower than that from the parameter identification of the MD system, cf. Figure 18. Furthermore, in order to get results comparable to those of the pure PS system, a Young's modulus of $E=800 \mathrm{MPa}$ is chosen which is slightly lower than that of the MD system. The displacements shown here are scaled by a factor of 15 . As in case of pure polystyrene, the deviations are close to zero at the $x z$ - and at the $y z$-surfaces, 
whereas its absolute values are larger at the $x z$-surfaces which are perpendicular to the load direction. Again, the stress distribution at these surfaces is not homogeneous which can most likely be traced back to the same reasons as discussed in Section 4.3. Furthermore, it is clearly visible at the surfaces perpendicular to the load direction that the absolute stress deviations increase with the load. The stress deviations are mainly negative which implies the particle domain being softer than the FE region, in particular for strains larger than three percent.

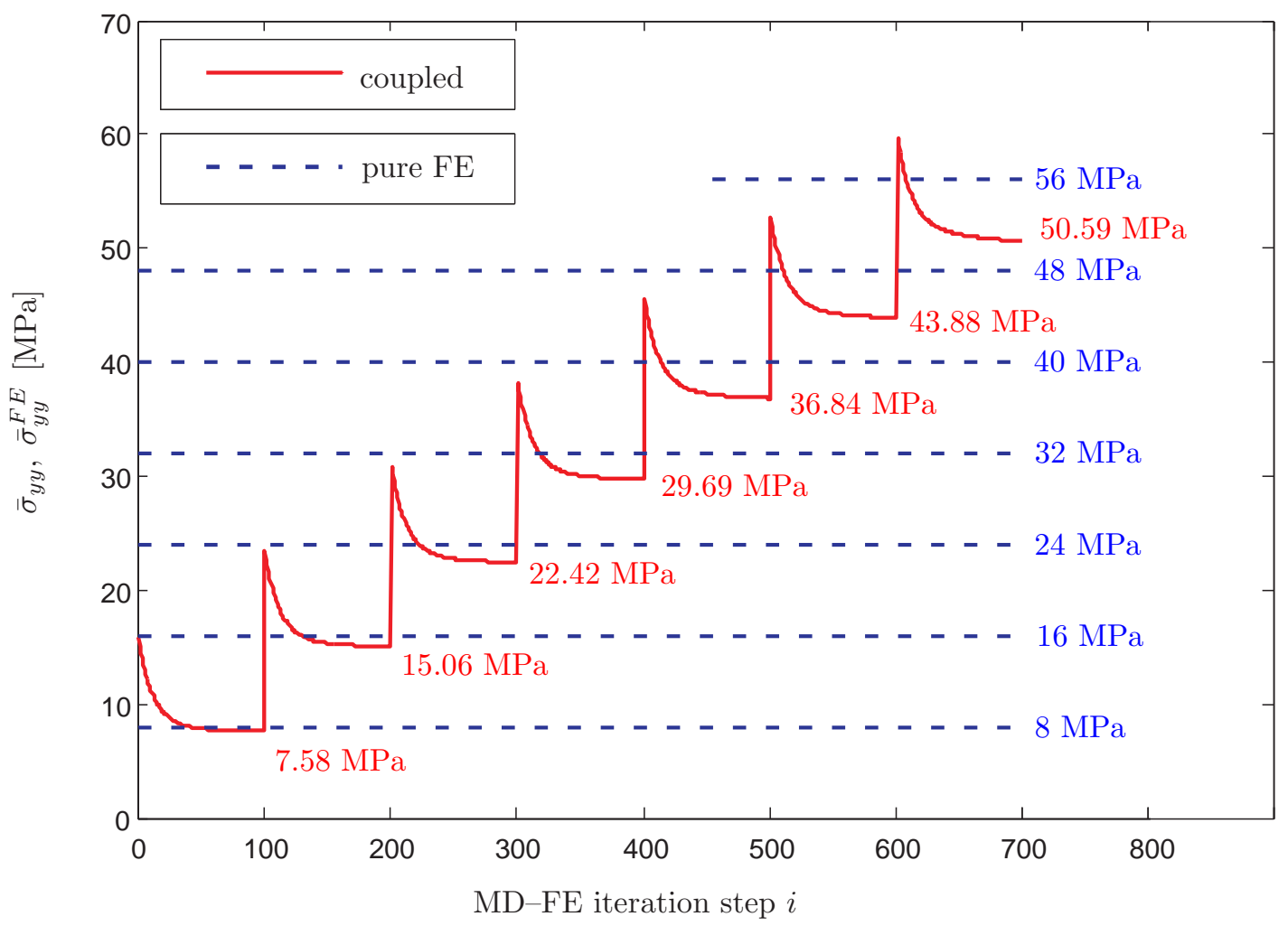

Figure 21: Coupled simulation with silica nanoparticle, seven loads steps, 1\% strain each, Young's modulus of the FE domain: $800 \mathrm{MPa}$; mean values of normal stress $\bar{\sigma}_{y y}$ (coupled nanocomposite) and $\bar{\sigma}_{y y}^{F E}$ (pure FE simulation) versus iteration steps, $100 \mathrm{MD}-\mathrm{FE}$ iterations equal one load step

This observation is quantified in Figure 21, the mean values of the tensile normal stress from the coupled and the FE reference simulation, $\bar{\sigma}_{y y}$ and $\bar{\sigma}_{y y}^{F E}$, are plotted versus iteration and load steps. It is clearly visible that the coupled simulation converges to approximately constant values of $\bar{\sigma}_{y y}$ within $100 \mathrm{MD}-\mathrm{FE}$ iterations for each load step. Thus, the system reaches an equilibrated configuration in each load step, which demonstrates that the coupling method is also able to treat polymer systems with filler particles. The absolute deviation between the results of the coupled and the reference simulation increases with the strain level, which is qualitatively evident also from Figure 20. This is not surprising since the strain considered here is surely beyond the linear elastic part of the stress-strain curve, cf. Figure 9. In order to capture the nonlinear behaviour of the particle system, the FE domain has to be extended to geometrical and physical nonlinearity, which is work in progress.

The curves plotted in Figure 21] are apparently very similar to those known from stress relaxation experiments of viscoelastic materials: the stress decreases when the specimen is subjected to constant strain. The conditions in the coupled simulation are similar: a certain strain is applied to the FE domain and kept constant during all MD-FE iteration steps within this load step. The MD domain has to follow this deformation, which is not instantly possible, but requires time for relaxation. It is not clear yet up to which part or whether at all the stress reduction observed in Figure 21 is caused simply by artefacts of the coupling procedure or if it results from the true viscoelasticity of the polystyrene reproduced by the MD. Possibly, the reason is a combination of both; however, careful and intense investigations are required to work out the effects taking place and thus to understand these findings. Though, this would again exceed the purpose of this work and has to be postponed to a later contribution.

A quantitative overview of the deviations and convergence criteria introduced in Section 4.2 is given 
in Table 2 which supports the qualitative observations. It is obvious that the deviation of stresses $e_{y y}^{\sigma}$ is much more sensitive to the mismatch between the coupled and the reference system. Compared to $e_{y}^{f}$, it renders a smaller deviation of $7.58 \%$ in the first load step, but it increases to $50.59 \%$ in the seventh load step which is about twice the value of $e_{y}^{f}$. Generally, all convergence criteria are significantly lower than $0.1 \%$ such that convergence can be assumed.

Table 2: Coupled simulation of polystyrene with nanoparticle: $e_{y}^{f}, e_{y y}^{\sigma}, \Delta e_{y(100)}^{f}, \Delta e_{y y}^{\sigma}(100)$, and $\Delta \bar{\sigma}_{y y(100)}$ after $100 \mathrm{MD}-\mathrm{FE}$ iterations per load step

\begin{tabular}{|r||r|r|r|r|r|r|r|}
\hline load step $i_{l s}$ & 1 & 2 & 3 & 4 & 5 & 6 & 7 \\
\hline \hline$e_{y(100)}^{f}[\%]$ & 12.16 & 13.79 & 15.63 & 17.24 & 19.07 & 20.97 & 24.09 \\
\hline$e_{y y(100)}^{\sigma}[\%]$ & 7.58 & 15.06 & 22.42 & 29.69 & 36.84 & 43.88 & 50.59 \\
\hline$\Delta e_{y(100)}^{f}[\%]$ & 0.0674 & 0.0413 & 0.0240 & 0.0305 & 0.0315 & 0.0301 & 0.0521 \\
\hline$\Delta e_{y y(100)}^{\sigma}[\%]$ & 0.0043 & 0.0075 & 0.0040 & 0.0130 & 0.0153 & 0.0177 & 0.0271 \\
\hline$\Delta \bar{\sigma}_{y y(100)}[\%]$ & -0.0283 & -0.0161 & -0.0089 & -0.0121 & -0.0120 & -0.0120 & -0.0186 \\
\hline
\end{tabular}

Figure 22 depicts the mean values of the normal stress after 100 MD-FE iterations per load step for the coupled simulation $\left(\bar{\sigma}_{y y(100)}\right)$ and for the FE simulation $\left(\bar{\sigma}_{y y(100)}^{F E}\right)$. From this plot, the nonlinear behaviour of the coupled system is clearly visible.

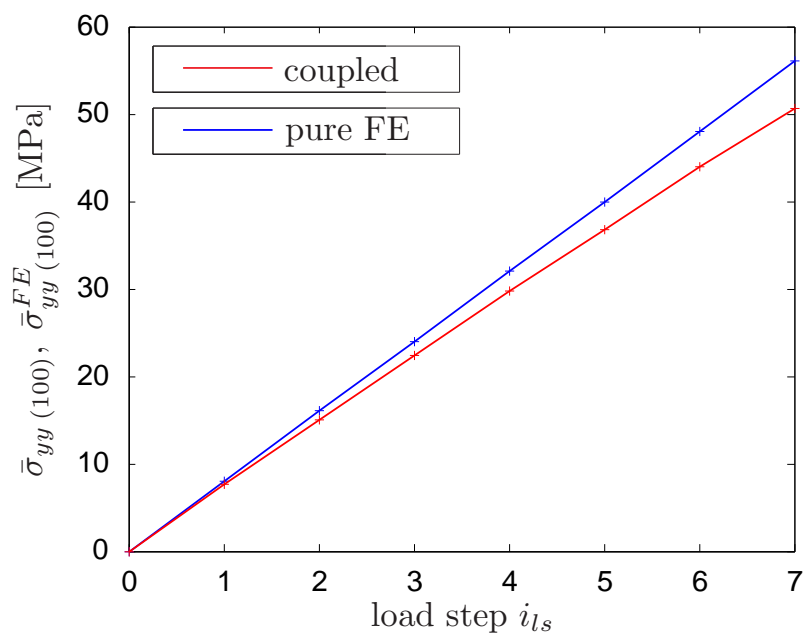

Figure 22: Comparison of the coupled nanocomposite and the pure FE simulation: mean values of the normal stress in load direction $\bar{\sigma}_{y y(100)}$ (coupled system) and $\bar{\sigma}_{y y(100)}^{F E}$ (pure FE system) after 100 MD-FE iterations for each load step versus load step $i_{l s}$; the Young's modulus is $E=800 \mathrm{MPa}$ and a strain of $1 \%$ is applied in each load step

In the subsequent section the results obtained so far are summarized and an outlook for future investigations is given.

\section{$5 \quad$ Summary and outlook}

A number of hybrid simulation techniques to couple a particle domain to a continuum has been developed in recent years. The large majority of these simulation procedures is however restricted to particle regions that are crystalline, e.g. [6]. The complexity of this type of hybrid simulations is largely simplified for the case of space-fixed particles. Coupled particle-continuum simulations for amorphous polymers, polymer nanocomposites, liquid crystals or liquids are still an exception. Recent hybrid simulation studies of these materials only allowed the coupling of particle domains which are mapped in different resolutions (atomistic versus coarse grained picture) 36]. This deficit in particle-continuum simulations for soft-matter systems motivated us to develop a hybrid technique allowing for a true 
coupling of continuum and particle domains with flexible atoms or CG beads. Such an approach would fill the gap between continuum models and particle approaches in new soft-matter materials such as nanocomposites which are in the focus of intensive academic and industrial research. To couple the two "worlds", a number of theoretical steps which are non-standard in both domains considered had to be implemented or even developed. For the description of the particle space a molecular dynamics formalism working with stochastic boundary conditions has been employed [22, 37. The conventional periodic boundary conditions adopted in the majority of MD simulations are incompatible with the spatial arrangement of the different domains of the coupled system. Nonperiodic stochastic boundary conditions have proven to be a suitable choice for the present hybrid approach.

To perform simulations of large particle domains, which is the final aim of the present long-term research, a coarse grained resolution has been chosen 18. Standard Newton's equations of motion in a classical MD description are solved in the inner core part of the coupled system which only contains the particles - in the present realization CG beads - of the soft-matter sample. Our test simulations have shown that the method works best when inserting a dissipative particle domain [22, [38] to dissipate energy and to control the temperature during the simulation. The spatial region surrounding the inner MD core and the DPD domain is the actual coupling region between particle and continuum description. The information transfer between both is provided by so-called anchor points, a set of auxiliary particles that are harmonically connected to the MD particles in the coupling region. The insertion of these anchor points has two advantages: on the one hand, the flexibly tethered MD particles perturb the MD particles in the core region less than strictly position-constrained particles. On the other hand, the harmonic description for the anchor point-MD particle interaction facilitates a fast calculation of force derivatives at the anchor points, a key ingredient of the proposed iterative coupling scheme. Forces and force gradients at the anchor points are transmitted from the particle to the continuum domain. The key parameters for the opposite information transfer are the anchor point positions which can change under the influence of the surrounding continuum being subjected to any Dirichlet or Neumann boundary conditions. The anchor point concept developed for the present hybrid method implies that the continuum is treated quasi-statically while the MD domain is dynamic.

The continuum is numerically solved by the Finite Element method and connected to the MD domain using the Arlequin approach [11, 12, [13. In order to solve the coupled system, a staggered iteration procedure has been set up together with an appropriate implementation of the information transfer between both domains. Test calculations on simple one-dimensional model systems 23 have shown possible staggered schemes to treat coupled systems. To blend the MD and FE energies in the coupling region several weighting functions have been suggested. The best choice, however, will require a careful optimization of the whole MD and FE parameter space in the present hybrid approach.

To verify the proposed method, we have performed coupled simulations on a sample of pure amorphous polystyrene as well as on a PS-silica nanocomposite. To quantify the accuracy of the coupling approach three different system descriptions have been correlated. First we have analyzed the pure PS sample in the framework of conventional MD simulations under periodic boundary conditions. These calculations provided a parameter identification, such as the evaluation of stress-strain curves and the calculation of Poisson's ratio under stress. The MD based parameters have been adopted for the second series of calculations, namely a FE simulation with input data from the particle domain.

Heart of the present study is, however, the performance of coupled MD-FE simulations. To estimate the accuracy and stability of the hybrid method several convergence criteria have been employed. Detailed discussions of the two deviations $e_{y}^{f}$ and $e_{y y}^{\sigma}$ of the reaction forces in the $y$-direction and of the Cauchy stress between a pure FE calculation and the coupled one have been given. Thereby we have verified that the stress in the coupling region is not homogeneously distributed as observed in the FE approach; it shows certain geometrical patterns. We are aware of the fact that an explanation of this phenomenon requires additional studies with the aim to optimize the design of the MD-FE coupling approach. This covers the spatial extension of the different domains, the number and position of the anchor points as well as the magnitude of the harmonic force constant between MD particles and anchor points.

Finally, plans for future research shall be mentioned. As it became obvious from this contribution, even nanocomposites can be simulated using the method developed here. Nevertheless, more complex systems containing multiple nanoparticles as well as different load cases will have to be investigated carefully. With the coupling scheme presented and a coarse grained description, system sizes should be in reach that are useful in engineering analyses on a macroscopic scale. Finally we hope that calculations of the present type will offer an access to continuum material parameters that can be justified on the basis of particle interactions. A first step into this direction has been made within the manuscript at 
hand.

We are indebted to Enrico Riccardi, Azadeh Ghanbari, and Tinashe Ndoro for useful discussions. Financial support by the European Union within the collaborative "Nanomodel" project (www.nanomodel.eu) and by the German Research Foundation (DFG) within the DFG-priority programme 1369 "Polymer-Solid Contacts: Interfaces and Interphases" (www.dfg-spp1369.de) is gratefully acknowledged.

\section{References}

[1] Daoulas KC, Müller M, Stoykovich MP, Park SM, Papakonstantopoulos YJ, de Pablo JJ, Nealey PF, Solak HH. Fabrication of complex three-dimensional nanostructures from self-assembling block copolymer materials on two-dimensional chemically patterned templates with mismatched symmetry. Physical Review Letters 2006; 96:036104.

[2] Daoulas KC, Müller M. Single chain in mean field simulations: Quasi-instantaneous field approximation and quantitative comparison with monte carlo simulations. The Journal of Chemical Physics 2006; 125:184904.

[3] Müller M, Smith GD. Phase separation in binary mixtures containing polymers: A quantitative comparison of single-chain-in-mean-field simulations and computer simulations of the corresponding multichain systems. Journal of Polymer Science Part B: Polymer Physics 2005; 43(8):934-958.

[4] Shilkrot LE, Curtin WA, Miller RE. A coupled atomistic/continuum model of defects in solids. Journal of the Mechanics and Physics of Solids 2002; 50(10):2085-2106.

[5] Shilkrot LE, Miller RE, Curtin WA. Multiscale plasticity modeling: coupled atomistics and discrete location mechanics. Journal of the Mechanics and Physics of Solids 2004; 52(4):755 - 787.

[6] Tadmor EB, Ortiz M, Phillips R. Quasicontinuum analysis of defects in solids. Philosophical Magazine A 1996; 73(6):1529-1563.

[7] Belytschko T, Xiao SP. Coupling methods for continuum model with molecular model. International Journal for Multiscale Computational Engineering 2003; 1:115-126.

[8] Guidault PA, Belytschko T. On the $l^{2}$ and $h^{1}$ couplings for an overlapping domain decomposition method using lagrange multipliers. International Journal for Numerical Methods in Engineering 2007; 70:322-350.

[9] Khare R, Mielke SL, Paci JT, Zhang S, Ballarini R, Schatz GC, Belytschko T. Coupled quantum mechanical/molecular mechanical modeling of the fracture of defective carbon nanotubes and graphene sheets. Physical Review B 2007; 75:075412.

[10] Zhang S, Khare R, Lu Q, Belytschko T. A bridging domain and strain computation method for coupled atomistic-continuum modelling of solids. International Journal for Numerical Methods in Engineering 2007; 70:913-933.

[11] Ben Dhia H. Problèmes méchaniques multi-échelles: la méthode arlequin. Comptes Rendus de l'Académie des Science, Series II b 1998; 326:899-904.

[12] Ben Dhia H, Rateau G. Analyse mathématique de la méthode arlequin mixte. Comptes Rendus de l'Académie des Science, Series I 2001; 332:649-654.

[13] Ben Dhia H, Rateau G. The arlequin method as a flexible engineering design tool. International Journal for Numerical Methods in Engineering 2005; 62:1442-1462.

[14] Bauman PT, Ben Dhia H, Elkhodja N, Oden JT, Prudhomme S. On the application of the arlequin method to the coupling of particle and continuum models. Computational Mechanics 2008; 42:511530. 
[15] Bauman PT, Oden JT, Prudhomme S. Adaptive multiscale modeling of polymeric materials with arlequin coupling and goals algorithms. Computer Methods in Applied Mechanics and Engineering $2009 ; 198: 799-818$.

[16] Chamoin L, Oden JT, Prudhomme S. A stochastic coupling method for atomic-to-continuum monte-carlo simulations. Computer Methods in Applied Mechanics and Engineering 2008; 197:3530-3546.

[17] Aubertin P, Rethore J, de Borst R. Energy conservation of atomistic/continuum coupling. International Journal for Numerical Methods in Engineering 2009; 78:1365-1386.

[18] Müller-Plathe F. Coarse-graining in polymer simulation: From the atomistic to the mesoscopic scale and back. ChemPhysChem 2002; 3(9):754-769.

[19] Müller-Plathe F. Scale-hopping in computer simulations of polymers. Soft Materials 2003; 1(1):131.

[20] Reith D, Pütz M, Müller-Plathe F. Deriving effective mesoscale potentials from atomistic simulations. Journal of Computational Chemistry 2003; 24(13):1624-1636.

[21] Milano G, Müller-Plathe F. Mapping atomistic simulations to mesoscopic models: A systematic coarse-graining procedure for vinyl polymer chains. The Journal of Physical Chemistry B 2005; 109(39):18609-18619.

[22] Rahimi M, Karimi-Varzaneh HA, Böhm MC, Müller-Plathe F, Pfaller S, Possart G, Steinmann P. Nonperiodic stochastic boundary conditions for molecular dynamics simulations of materials embedded into a continuum domain. Journal of Chemical Physics 2011; 134:154108.

[23] Pfaller S, Possart G, Steinmann P, Rahimi M, Müller-Plathe F, Böhm MC. A comparison of staggered solution schemes for coupled particle-continuum systems modeled with the arlequin method. Computational Mechanics 2012; 49:565-579.

[24] Qian H, Carbone P, Chen X, Karimi-Varzaneh HA, Müller-Plathe F. Temperature-transferable coarse-grained potentials for ethylbenzene, polystyrene, and their mixtures. Macromolecules 2008; 41(24):9919-9929.

[25] Ghanbari A, Rahimi M, Böhm MC, Müller-Plathe F. Coarse grained molecular dynamics simulation of polystyrene-silica nanocomposite: Dynamics in the interphase and polymer-mediated interactions of nanoparticles. Journal of Physical Chemistry $C$; submitted.

[26] Karimi-Varzaneh HA, Qian HJ, Chen X, Carbone P, Müller-Plathe F. Ibisco: A molecular dynamics simulation package for coarse-grained simulation. Journal of Computational Chemistry 2011; 32:1475-1487.

[27] Español P, Warren P. Statistical mechanics of dissipative particle dynamics. Europhysics Letters 1995; 30:191.

[28] Koelman JMVA, Hoogerbrugge PJ. Dynamic simulations of hard-sphere suspensions under steady shear. Europhysics Letters 1993; 21:363.

[29] Delgado-Buscalioni R, Kremer K, Praprotnik M. Concurrent triple-scale simulation of molecular liquids. The Journal of Chemical Physics 2008; 128:114110.

[30] Belytschko T, Krongauz Y, Organ D, Fleming M, Krysl P. Meshless methods: An overview and recent developments. Computer Methods in Applied Mechanics and Engineering 1996; 139:3-47.

[31] Rahimi M, Iriarte-Carretero I, Ghanbari A, Böhm MC, Müller-Plathe F. Mechanical behavior and interphase structure in a silica-polystyrene nanocomposite under uniaxial deformation. Nanotechnology 2012; 23:305 702.

[32] Lyulin AV, Balabaev NK, Mazo MA, Michels MAJ. Molecular dynamics simulation of uniaxial deformation of glassy amorphous atactic polystyrene. Macromolecules 2004; 37(23):8785-8793.

[33] Heinrich G, Vilgis TA. Contribution of entanglements to the mechanical properties of carbon blackfilled polymer networks. Macromolecules 1993; 26(5):1109-1119. 
[34] Everaers R, Kremer K. Elastic properties of polymer networks. Journal of Molecular Modeling ; 2:293-299.

[35] Ghanbari A, Ndoro TVM, Leroy F, Rahimi M, Böhm MC, Müller-Plathe F. Interphase structure in silica-polystyrene nanocomposites: A coarse-grained molecular dynamics study. Macromolecules 2011; 45:572-584.

[36] Abrams CF, Delle Site L, Kremer K. Dual-resolution coarse-grained simulation of the bisphenola-polycarbonate/nickel interface. Physical Review E Feb 2003; 67:021 807.

[37] Allen P, Tildesley D. Computer Simulation of Liquids. Oxford Science Publications, Clarendon Press, 1987.

[38] Qian HJ, Liew CC, Müller-Plathe F. Effective control of the transport coefficients of a coarsegrained liquid and polymer models using the dissipative particle dynamics and lowe-andersen equations of motion. Phys. Chem. Chem. Phys. 2009; 11:1962. 